\title{
On the isomorphism classes of weighted spaces of harmonic and holomorphic functions
}

\author{
by \\ WolfGang Lusky (Paderborn)
}

\begin{abstract}
Let $\Omega$ be either the complex plane or the open unit disc. We completely determine the isomorphism classes of

$$
H v=\left\{f: \Omega \rightarrow \mathbb{C} \text { holomorphic } \sup _{z \in \Omega}|f(z)| v(z)<\infty\right\}
$$

and investigate some isomorphism classes of

$$
h v=\left\{f: \Omega \rightarrow \mathbb{C} \text { harmonic } \sup _{z \in \Omega}|f(z)| v(z)<\infty\right\}
$$

where $v$ is a given radial weight function. Our main results show that, without any further condition on $v$, there are only two possibilities for $H v$, namely either $H v \sim l_{\infty}$ or $H v \sim$ $H_{\infty}$, and at least two possibilities for $h v$, again $h v \sim l_{\infty}$ and $h v \sim H_{\infty}$. We also discuss many new examples of weights.
\end{abstract}

1. Introduction. Fix $a>0$ or $a=\infty$ and put $a D=\{z \in \mathbb{C}:|z|<a\}$ (i.e. $a D=\mathbb{C}$ if $a=\infty$ ). For $0<r<a$ and $f: a D \rightarrow \mathbb{C}$ put $M_{\infty}(f, r)=$ $\sup _{|z|=r}|f(z)|$. Recall that $M_{\infty}(f, r)$ is increasing with respect to $r$ if $f$ is a harmonic function ([5]).

We want to investigate spaces of harmonic and holomorphic functions $f$ where $M_{\infty}(f, r)$ is unbounded in general but grows in a controlled way. To this end we introduce a weight function, i.e. an upper semicontinuous, non-increasing function $v:\left[0, a[\rightarrow] 0, \infty\left[\right.\right.$ with $\lim _{r \rightarrow a} r^{m} v(r)=0$ for all $m \geq 0$. (If $a<\infty$ this is equivalent to $\lim _{r \rightarrow a} v(r)=0$.) We study the growth conditions

$$
M_{\infty}(f, r)=O\left(\frac{1}{v(r)}\right) \text { and } M_{\infty}(f, r)=o\left(\frac{1}{v(r)}\right) \quad \text { as } r \rightarrow a
$$

by defining $\|f\|_{v}=\sup _{z \in a D}|f(z)| v(|z|)$ and

$$
h v=\left\{f: a D \rightarrow \mathbb{C} \text { harmonic }:\|f\|_{v}<\infty\right\},
$$

2000 Mathematics Subject Classification: Primary 46E15; Secondary 46B03.

Key words and phrases: holomorphic functions, harmonic functions, weighted spaces.

This research was supported by DFG, No. LU 219/7-2. 


$$
\begin{aligned}
(h v)_{0} & =\left\{f \in h v: \lim _{r \rightarrow a} M_{\infty}(f, r) v(r)=0\right\}, \\
H v & =\{f \in h v: f \text { holomorphic }\}, \\
(H v)_{0} & =(H v) \cap(h v)_{0} .
\end{aligned}
$$

These are Banach spaces (with respect to $\|\cdot\|_{v}$ ). The condition on $v$ ensures that these spaces contain all polynomials (or trigonometric polynomials, resp.). For example, if $f: a D \rightarrow \mathbb{C}$ is harmonic, then clearly

$$
M_{\infty}(f, r)=O\left(\frac{1}{v(r)}\right) \text { as } r \rightarrow a \text { if and only if } f \in h v
$$

and

$$
M_{\infty}(f, r)=o\left(\frac{1}{v(r)}\right) \text { as } r \rightarrow a \quad \text { if and only if } f \in(h v)_{0} .
$$

By a simple substitution argument we see that it suffices to consider the two cases $a=1$ and $a=\infty$. We want to discuss the Banach space nature of $h v,(h v)_{0}, H v$ and $(H v)_{0}$. In this respect a lot has already been done for holomorphic and harmonic functions on the unit disc where $v$ is a moderately decreasing weight $([10,14,16,19-21]$; see also $[2,3,6,7,17])$. But only few results are known for fast decreasing weights and for functions on the complex plane $([8,9])$.

In this article we determine all possible isomorphism classes for $H v$ and $(H v)_{0}$ and some isomorphism classes for $h v$ and $(h v)_{0}$ without any further condition on $v$.

Let $v:\left[0, a\left[\rightarrow \mathbb{R}_{+}\right.\right.$be a weight function. For $m>0$ fix a global maximum point $r_{m}$ of the function $r \mapsto r^{m} v(r), r \in[0, a$ [, which exists in view of the upper semicontinuity. It is easily seen that $r_{m} \uparrow a$ as $m \rightarrow \infty$, and $m \mapsto$ $r_{m}^{m} v\left(r_{m}\right), m>0$, is a continuous function. We want to compare quotients of the form $\left(r_{m} / r_{n}\right)^{m} v\left(r_{m}\right) / v\left(r_{n}\right)$ for different $m$ and $n$. First we introduce the following boundedness condition on $v$ :

$$
\begin{aligned}
& \forall b_{1}>1 \exists b_{2}>1 \exists c>0 \forall m, n>0: \\
& \left(\frac{r_{m}}{r_{n}}\right)^{m} \frac{v\left(r_{m}\right)}{v\left(r_{n}\right)} \leq b_{1} \text { and } m, n,|m-n| \geq c \Rightarrow\left(\frac{r_{n}}{r_{m}}\right)^{n} \frac{v\left(r_{n}\right)}{v\left(r_{m}\right)} \leq b_{2} .
\end{aligned}
$$

Examples of $v$ enjoying (B) include $(1-r)^{\alpha}$ for $\alpha>0, \exp \left(-(1-r)^{-1}\right)$, $\exp \left(-\exp \left((1-r)^{-1}\right)\right), \ldots$, if $r \in\left[0,1\left[\right.\right.$, and $\exp \left(-r^{\varrho}\right)$ for $\varrho>0, \exp \left(-\log ^{\gamma} r\right)$ for $\gamma \geq 2, \exp (-\exp (r)), \exp (-\exp (\exp (r))), \ldots$ if $r \in \mathbb{R}_{+}$(see the next section for details).

Observe that the negation of (B) reads as follows:

$\neg$ (B) $\exists b_{1}>1 \forall b_{2}>1 \forall c>0 \exists m, n>0$ :

$$
\left(\frac{r_{m}}{r_{n}}\right)^{m} \frac{v\left(r_{m}\right)}{v\left(r_{n}\right)} \leq b_{1} \text { and } m, n,|m-n| \geq c \text { and }\left(\frac{r_{n}}{r_{m}}\right)^{n} \frac{v\left(r_{n}\right)}{v\left(r_{m}\right)} \geq b_{2} \text {. }
$$


For two Banach spaces $X$ and $Y$ we write $X \sim Y$ if they are isomorphic to each other. Let $d(X, Y)$ be the Banach-Mazur distance of $X$ and $Y$, i.e.

$$
d(X, Y)=\inf \left\{\|T\| \cdot\left\|T^{-1}\right\|: T: X \rightarrow Y \text { is an (onto) isomorphism }\right\} .
$$

Let $H_{n}=\operatorname{span}\left\{1, z^{1}, z^{2}, \ldots, z^{n}\right\}$ be the space of functions on $\partial D$ with the norm $M_{\infty}(\cdot, 1)$. It is well known that the Hardy space

$$
H_{\infty}=\left\{f: D \rightarrow \mathbb{C}: f \text { holomorphic, } \sup _{0<r<1} M_{\infty}(f, r)<\infty\right\}
$$

is isomorphic to $\left(\sum_{n} \oplus H_{n}\right)_{\infty}([22])$.

\subsection{THEOREM.}

(a) Let $v$ satisfy $(\mathrm{B})$. Then $H v \sim l_{\infty}$ and $(H v)_{0} \sim c_{0}$.

(b) Let $v$ satisfy $\neg(\mathrm{B})$. Then $H v \sim H_{\infty}$ and $(H v)_{0} \sim\left(\sum_{n} \oplus H_{n}\right)_{0}$.

Sections 3-6 are dedicated to the proofs of Theorem 1.1 and the following results.

For the isomorphic classification of $h v$ we need another boundedness condition:

(C) $\exists c_{1}>0 \exists b_{1}>1 \forall b_{2}>1 \forall c_{2}>0 \exists m, n>0$ :

$$
\begin{aligned}
& \left(\frac{r_{n}}{r_{m}}\right)^{n} \frac{v\left(r_{n}\right)}{v\left(r_{m}\right)} \leq b_{1}, \quad\left(\frac{r_{m}}{r_{n}}\right)^{m} \frac{v\left(r_{m}\right)}{v\left(r_{n}\right)} \geq b_{2}, \\
& m, n,|n-m| \geq c_{2} \quad \text { and } \quad c_{1}|n-m|<\min (m, n) .
\end{aligned}
$$

Observe that $(\mathrm{C}) \Rightarrow \neg(\mathrm{B})$.

\subsection{THEOREM.}

(a) If $v$ satisfies $(\mathrm{B})$ then $h v \sim l_{\infty}$ and $(h v)_{0} \sim c_{0}$.

(b) If $v$ satisfies $(\mathrm{C})$ then $h v \sim H_{\infty}$ and $(h v)_{0} \sim\left(\sum_{n} \oplus H_{n}\right)_{0}$.

If $v$ satisfies (C) then we have the combination $h v \sim H v \sim H_{\infty}$ while (B) implies $h v \sim H v \sim l_{\infty}$. If $H v \sim l_{\infty}$ then it is easily seen that $h v \sim H_{v} \oplus H_{v}$ and hence also $h v \sim l_{\infty}$. However, we can also have the combination $H v \sim$ $H_{\infty}$ and $h v \sim l_{\infty}$ (see the following example). It is likely that these three are the only possibilities.

EXAMPLE. Let $v(r)=(1-\log (1-r))^{-1}, r \in[0,1[$. It is known that here $H v \sim H_{\infty}$ and $h v \sim l_{\infty}([10,16])$. Hence $v$ satisfies $\neg(\mathrm{B})$ and $\neg(\mathrm{C})$.

We also investigate under which (sufficient) condition $h v$ is selfadjoint, i.e. we have $f \in h v$ if and only if $\widetilde{f} \in h v$ where $\widetilde{f}$ is the trigonometric conjugate of $f$. $(\tilde{f}$ is such that $\widetilde{f}(0)=0$ and $\operatorname{Re} f+i \operatorname{Re} \widetilde{f}, \operatorname{Im} f+i \operatorname{Im} \widetilde{f}$ are holomorphic.) This is equivalent to the fact that the Riesz projection $R: h v \rightarrow H v$ with

$$
R\left(r^{|k|} \exp (i k \varphi)\right)=\left\{\begin{array}{ll}
r^{k} \exp (i k \varphi), & k \geq 0, \\
0, & \text { else }
\end{array} \quad k \in \mathbb{Z},\right.
$$


is bounded. (We frequently denote the $k$ th monomials on $\mathbb{C}$ by $z^{k}, \bar{z}^{k}$ or $r^{k} \exp (i k \varphi), r^{|k|} \exp (-i k \varphi)$.) We have $\widetilde{f}=-i R f+i(\mathrm{id}-R) f+i f(0)$.

1.3. Theorem. Let $v$ satisfy (B). Then $h v$ is selfadjoint.

Hence, in particular, a harmonic function $f$ satisfies

$$
M_{\infty}(f, r)=O\left(\frac{1}{v(r)}\right) \text { as } r \rightarrow a \quad \text { if and only if } \quad M_{\infty}(\tilde{f}, r)=O\left(\frac{1}{v(r)}\right) .
$$

(B) is a condition about a certain "inner regularity" of $v$ rather than its decay. To give a geometrical interpretation of $(\mathrm{B})$ put $\varphi(t)=-\log \left(v\left(e^{t}\right)\right)$, where $t \in]-\infty, 0[$ if $a=1$ and $t \in \mathbb{R}$ if $a=\infty$. Then $v(r)=\exp (-\varphi(\log r))$. The conditions on $v$ imply that $\varphi$ is increasing and that $\varphi(t) \rightarrow \infty$ as $t \rightarrow 0$ for $a=1$, and $\varphi(t) / t \rightarrow \infty$ as $t \rightarrow \infty$ for $a=\infty$. Due to Hadamard's three circles theorem we may change $v$ on bounded annuli without changing the isomorphic character of $H v,(H v)_{0}, h v$ or $(h v)_{0}$. Therefore we may assume without loss of generality that $\varphi$ is twice differentiable. The function $r \mapsto r^{m} v(r)$ has a maximum only if $\varphi^{\prime}(\log r)=m$. Put $s=\log r_{m}$ and $t=\log r_{n}$. Then we have

$$
\log \left(\left(\frac{r_{m}}{r_{n}}\right)^{m} \frac{v\left(r_{m}\right)}{v\left(r_{n}\right)}\right)=\varphi(t)-\varphi(s)-\varphi^{\prime}(s)(t-s)=: \varrho(t, s) ;
$$

$\varrho(t, s)$ is the distance between the graph of $\varphi$ and its tangent.

Now, (B) is equivalent to the following

$$
\begin{aligned}
\forall b_{1}>0 \exists b_{2} & >0 \exists c>0 \forall s, t: \\
\varrho(t, s) & \leq b_{1},\left|\varphi^{\prime}(t)\right|,\left|\varphi^{\prime}(s)\right|,\left|\varphi^{\prime}(t)-\varphi^{\prime}(s)\right| \geq c \Rightarrow \varrho(s, t) \leq b_{2} .
\end{aligned}
$$

This means that the graph of $\varphi$ has no big corners. (See also the remark following Example 2.4.)

Acknowledgements. I am indebted to the referee for many valuable remarks. In particular the preceding geometric interpretation of condition (B) is due to him.

2. More examples. Here we give several examples where (B) holds.

2.1. ExAmple. $v(r)=\exp (-\exp (r)), r \in\left[0, \infty\left[\right.\right.$. Then $r_{n \log n}=\log n$ for any $n>0$. Fix $m, n>0$. For $m^{\prime}=m \log m$ and $n^{\prime}=n \log n$ we obtain

$$
\begin{aligned}
\left(\frac{r_{m^{\prime}}}{r_{n^{\prime}}}\right)^{m^{\prime}} \frac{v\left(r_{m^{\prime}}\right)}{v\left(r_{n^{\prime}}\right)} & =\exp (m \log m(\log \log m-\log \log n)+n-m) \\
& =\exp \left(\frac{(n-m)^{2}(m \log m)(1+\log \bar{m})}{2 \bar{m}^{2} \log ^{2} \bar{m}}\right)
\end{aligned}
$$


for some $\bar{m}$ between $m$ and $n$. (We have used

$$
\log \log n-\log \log m=\frac{n-m}{m \log m}-\frac{1+\log \bar{m}}{2(\bar{m} \log \bar{m})^{2}}(n-m)^{2}
$$

for appropriate $\bar{m}$.) Moreover the function

$$
\left.n \mapsto\left(\frac{r_{m^{\prime}}}{r_{n \log n}}\right)^{m^{\prime}} \frac{v\left(r_{m^{\prime}}\right)}{v\left(r_{n \log n}\right)}, \quad n>0 \text { (for fixed } m\right),
$$

is increasing if $n>m$ and decreasing if $n<m$.

Fix $b_{1}>1$ and put $\beta=4 \sqrt{\log b_{1}}, c=\max \left(64 \log b_{1}, 2\right)$. Hence, if $m \geq c$ then $\beta / \sqrt{m} \leq 1 / 2$. If $|n-m|=\beta \sqrt{m}, n, m \geq c$, then we obtain

$$
\begin{aligned}
\left(\frac{r_{m^{\prime}}}{r_{n^{\prime}}}\right)^{m^{\prime}} \frac{v\left(r_{m^{\prime}}\right)}{v\left(r_{n^{\prime}}\right)} & \geq \exp \left(\frac{\beta^{2} m^{2}(\log m)(1+\log \bar{m})}{2 \bar{m}^{2} \log ^{2} \bar{m}}\right) \\
& \geq \exp \left(\beta^{2} \frac{1}{2}\left(\frac{m}{\bar{m}}\right)^{2} \frac{\log m}{\log \bar{m}}\right) \\
& \geq \exp \left(\beta^{2} \frac{1}{2}\left(\frac{1}{1+\beta / \sqrt{m}}\right)^{2} \frac{\log m}{\log m+\log (1+\beta / \sqrt{m})}\right) \\
& \geq \exp \left(\frac{\beta^{2}}{16}\right)=b_{1} .
\end{aligned}
$$

This implies that $|n-m| \leq \beta \sqrt{m}$ whenever

$$
\left(\frac{r_{m^{\prime}}}{r_{n^{\prime}}}\right)^{m^{\prime}} \frac{v\left(r_{m^{\prime}}\right)}{v\left(r_{n^{\prime}}\right)} \leq b_{1} .
$$

In this case we have

$$
\begin{aligned}
\left(\frac{r_{n^{\prime}}}{r_{m^{\prime}}}\right)^{n^{\prime}} \frac{v\left(r_{n^{\prime}}\right)}{v\left(r_{m^{\prime}}\right)} & =\exp \left(\frac{(n-m)^{2}(n \log n)(1+\log \bar{n})}{2 \bar{n}^{2} \log ^{2} \bar{n}}\right) \\
& \leq \exp \left(\frac{(n-m)^{2} n \log n}{\bar{n}^{2} \log \bar{n}}\right) \\
& \leq \exp \left(\beta^{2} m \frac{(m+\beta \sqrt{m}) \log (m+\beta \sqrt{m})}{(m-\beta \sqrt{m})^{2} \log (m-\beta \sqrt{m})}\right) \\
& \leq \exp \left(\beta^{2} \frac{(1+\beta / \sqrt{m})(\log m+\log (1+\beta / \sqrt{m}))}{(1-\beta / \sqrt{m})^{2}(\log m+\log (1-\beta / \sqrt{m}))}\right) \leq b_{2}
\end{aligned}
$$

for suitable $b_{2}$ independent of $m$. (Here $\bar{n}$ is an appropriate number between $m$ and $n$.) Thus $v$ satisfies (B). Similarly one can deal with $\exp \left(-r^{\varrho}\right)$ for $\varrho>0, \exp (-\exp (\exp (r))), \ldots$

2.2. ExAmple. $v(r)=\exp \left(-\log ^{\varrho} r\right), r \in[1, \infty[$, for fixed $\varrho \geq 2$, and $v(r)=1, r \in\left[0,1\left[\right.\right.$. Here we obtain $r_{n}=\exp \left((n / \varrho)^{1 /(\varrho-1)}\right)$ (for sufficiently 
large $n)$. We have

$$
\begin{aligned}
\left(\frac{r_{m}}{r_{n}}\right)^{m} & \frac{v\left(r_{m}\right)}{v\left(r_{n}\right)} \\
& =\exp \left((\varrho-1)\left(\left(\frac{m}{\varrho}\right)^{\frac{\varrho}{\varrho-1}}-\left(\frac{n}{\varrho}\right)^{\frac{\varrho}{\varrho-1}}\right)+(n-m)\left(\frac{n}{\varrho}\right)^{\frac{1}{\varrho-1}}\right) \\
& =\exp \left(\frac{(n-m)^{2}}{2(\varrho-1) \varrho^{\frac{1}{\varrho-1}} \bar{m}^{\frac{\varrho-2}{\varrho-1}}}\right)
\end{aligned}
$$

for suitable $\bar{m}$ between $m$ and $n$. (We used

$$
x^{\beta}-x_{0}^{\beta}=\beta x_{0}^{\beta-1}\left(x-x_{0}\right)+\frac{1}{2} \beta(\beta-1) \bar{x}^{\beta-2}\left(x-x_{0}\right)^{2}
$$

for $x=m / \varrho, x_{0}=n / \varrho, \beta=\varrho /(\varrho-1)$ and appropriate $\bar{x}$.) The map

$$
n \mapsto\left(\frac{r_{m}}{r_{n}}\right)^{m} \frac{v\left(r_{m}\right)}{v\left(r_{n}\right)}
$$

is increasing if $n>m$ and decreasing if $n<m$ (for fixed $m$ ). Fix $b_{1}>1$ and put

$$
\gamma=\frac{\varrho-2}{\varrho-1}, \quad \beta=\sqrt{2^{\gamma+1}(\varrho-1) \varrho^{1 /(\varrho-1)} \log b_{1}}, \quad c=(2 \beta)^{2(\varrho-1) / \varrho} .
$$

Then $\beta m^{\gamma / 2-1} \leq 1 / 2$ provided that $m \geq c$. If $|n-m|=\beta m^{\gamma / 2}$ and $n, m \geq c$ we obtain

$$
\left(\frac{r_{m}}{r_{n}}\right)^{m} \frac{v\left(r_{m}\right)}{v\left(r_{n}\right)} \geq \exp \left(2^{\gamma}\left(\log b_{1}\right)\left(\frac{m}{m+\beta m^{\gamma / 2}}\right)^{\gamma}\right) \geq b_{1} .
$$

Hence, if

$$
\left(\frac{r_{m}}{r_{n}}\right)^{m} \frac{v\left(r_{m}\right)}{v\left(r_{n}\right)} \leq b_{1}
$$

then $|n-m| \leq \beta m^{\gamma / 2}$ and

$$
\begin{aligned}
\left(\frac{r_{n}}{r_{m}}\right) \frac{v\left(r_{m}\right)}{v\left(r_{n}\right)} & =\exp \left(\frac{(n-m)^{2}}{2(\varrho-1) \varrho^{\frac{1}{\varrho-1}} \bar{n}^{\frac{\varrho-2}{\varrho-1}}}\right) \\
& \leq \exp \left(2^{\gamma}\left(\frac{m}{m-\beta m^{\gamma / 2}}\right)^{\gamma} \log b_{1}\right) \leq b_{1}^{4^{\gamma}}=: b_{2}
\end{aligned}
$$

(for suitable $\bar{n}$ between $m$ and $n$ ).

2.3. ExAmple. $v(r)=\exp (-1 /(1-r)), r \in\left[0,1\left[\right.\right.$. Here $r_{m^{2}-m}=1-$ $1 / m$. Fix $m, n>0$. For $m^{\prime}=m^{2}-m$ and $n^{\prime}=n^{2}-n$ we obtain

$$
\left(\frac{r_{m^{\prime}}}{r_{n^{\prime}}}\right)^{m^{\prime}} \frac{v\left(r_{m^{\prime}}\right)}{v\left(r_{n^{\prime}}\right)}=\left(\frac{1-\frac{1}{m}}{1-\frac{1}{n}}\right)^{m^{2}-m} \exp (n-m) .
$$


Hence

$$
n \mapsto\left(\frac{r_{m^{\prime}}}{r_{n^{2}-n}}\right)^{m^{\prime}} \frac{v\left(r_{m^{\prime}}\right)}{v\left(r_{n^{2}-n}\right)}
$$

is decreasing if $n<m$ and increasing if $n>m$. Fix $\beta>0$ and put

$$
a_{m}=\left(\frac{1-\frac{1}{m}}{1-\frac{1}{m \pm \beta \sqrt{m}}}\right)^{m^{2}-m} \exp ( \pm \beta \sqrt{m}) \text {. }
$$

We obtain $\lim _{m \rightarrow \infty} a_{m}=\exp \left(\beta^{2}\right)$. Define $\beta=\sqrt{2 \log b_{1}}$ and take $c$ so large that $a_{m} \geq \exp \left(\log b_{1}\right)=b_{1}$ whenever $m \geq c$. Thus, if $|n-m|=\beta \sqrt{m}$ we have

$$
\left(\frac{r_{m^{\prime}}}{r_{n^{\prime}}}\right)^{m^{\prime}} \frac{v\left(r_{m^{\prime}}\right)}{v\left(r_{n^{\prime}}\right)} \geq b_{1} .
$$

So, if

$$
\left(\frac{r_{m^{\prime}}}{r_{n^{\prime}}}\right)^{m^{\prime}} \frac{v\left(r_{m^{\prime}}\right)}{v\left(r_{n^{\prime}}\right)} \leq b_{1}
$$

we must have $|n-m| \leq \beta \sqrt{m}$. In this case we obtain

$$
\begin{aligned}
\left(\frac{r_{n^{\prime}}}{r_{m^{\prime}}}\right)^{n^{\prime}} \frac{v\left(r_{n^{\prime}}\right)}{v\left(r_{m^{\prime}}\right)} & =\left(\frac{1-\frac{1}{n}}{1-\frac{1}{m}}\right)^{n^{2}-n} \exp (m-n) \\
& =\left(1+\frac{n-m}{m-1} \cdot \frac{1}{n}\right)^{n^{2}-n} \exp (m-n) \\
& \leq \exp \left(\frac{(n-m)^{2}}{m-1}\right) \leq \exp \left(2 \beta^{2}\right)=: b_{2} .
\end{aligned}
$$

Similarly one can show that $\exp (-\exp (1 /(1-r))), \exp (-\exp (\exp (1 /(1-r))))$, ... satisfy $(\mathrm{B})$.

2.4. Example. $v(r)=(1-r)^{\alpha}, r \in[0,1[$, for some fixed $\alpha>0$. Here $r_{n}=n /(n+\alpha)$ and, as in the preceding example, we can verify that $v$ satisfies $(\mathrm{B})$.

The weight of Example 2.4 is of moderate decay, it satisfies

$$
\sup _{n} \frac{v\left(1-2^{-n}\right)}{v\left(1-2^{-n-1}\right)}<\infty .
$$

Such weights have been studied extensively. Here it is possible to fix $m_{1}<$ $m_{2}<\cdots$ and $\gamma>1$ such that

$$
\gamma \leq \frac{v\left(1-2^{-m_{n}}\right)}{v\left(1-2^{\left.-m_{n+1}\right)}\right.} \leq \gamma^{2} \quad \text { for all } n
$$

This implies the existence of an index $j$ with $1-\frac{1}{2^{m_{n-j}}} \leq r_{M} \leq 1-\frac{1}{2^{m_{n+j}}} \quad$ whenever $\quad 2^{m_{n}} \leq M<2^{m_{n+1}}, \quad n=1,2, \ldots$ 
Using this one can show that condition (B) is equivalent to

$$
\inf _{k} \limsup _{n} \frac{v\left(1-2^{-n-k}\right)}{v\left(1-2^{-n}\right)}<1
$$

provided that $(\star)$ holds. Hence Theorem 1.1 includes one of the main results of [16]. (We omit the details.) Weights satisfying $(\star)$ and $(\star \star)$ are called normal (see [4], [13], [19]-[21]).

The following proposition allows us to construct examples for all the cases discussed in Section 1.

2.5. Proposition. Fix numbers $1 \leq n_{1}<n_{2}<\cdots, 0<s_{1}<s_{2}<\cdots$ and $v_{1}>v_{2}>\cdots>0$ such that $\sup _{k} n_{k}<\infty, \lim _{k \rightarrow \infty} s_{k}=a$ and

$$
\begin{aligned}
s_{m}^{n_{m}} v_{m} & =\sup _{k} s_{k}^{n_{m}} v_{k}, \\
\lim _{k \rightarrow \infty} s_{k}^{n_{m}} v_{k} & =0 \quad \text { for each } m .
\end{aligned}
$$

Put $v(s)=v_{m}$ if $s_{m-1}<s \leq s_{m}$. Then $v$ is a weight on $\left[0, a\left[\right.\right.$ with $r_{n_{m}}=s_{m}$ for all $m$. Moreover, if $n_{m-1}<j<n_{m}$ then

$$
r_{j}= \begin{cases}s_{m-1} & \text { if } s_{m-1}^{j} v_{m-1} \geq s_{m}^{j} v_{m} \\ s_{m} & \text { else. }\end{cases}
$$

Proof. $v$ is upper semicontinuous, non-increasing and $\lim _{r \rightarrow a} r^{m} v(r)=0$ for all $m \geq 0$. Fix $m$. If $s_{k-1}<s \leq s_{k}$ then $s^{n_{m}} v(s)=s^{n_{m}} v_{k} \leq s_{k}^{n_{m}} v_{k} \leq$ $s_{m}^{n_{m}} v_{m}$. Hence $r_{n_{m}}=s_{m}$.

Now, let $n_{m-1}<j<n_{m}$. If $k \leq m-1$ and $s_{k-1}<s \leq s_{k}$ then

$$
s^{j} v(s) \leq s_{k}^{j} v_{k} \leq s_{k}^{j-n_{m-1}} s_{m-1}^{n_{m-1}} v_{m-1} \leq s_{m-1}^{j} v_{m-1} .
$$

If $k \geq m$ and $s_{k}<s \leq s_{k+1}$ then

$$
s^{j} v(s) \leq s^{j-n_{m}} s_{m}^{n_{m}} v_{m} \leq s_{m}^{j} v_{m} .
$$

Finally, if $s_{m-1}<s \leq s_{m}$ then $s^{j} v(s)=s^{j} v_{m} \leq s_{m}^{j} v_{m}$. Hence $r_{j}=s_{m-1}$ if $s_{m-1}^{j} v_{m-1} \geq s_{m}^{j} v_{m}$, and $r_{j}=s_{m}$ otherwise.

2.6. Example. Using Proposition 2.5 we construct a weight $v$ on $[0, \infty[$ which satisfies $(\mathrm{C})$. To this end put

$$
s_{m}=m !, \quad n_{m}=\sum_{j=1}^{m} j, \quad v_{m}=\prod_{j=1}^{m} \frac{1}{j^{n_{j}}} .
$$

Then $s_{m}^{n_{m}} v_{m}=\prod_{j=1}^{m} j^{n_{m}-n_{j}}$. Moreover 


$$
s_{k}^{n_{m}} v_{k}= \begin{cases}\prod_{j=1}^{k} j^{n_{m}-n_{j}} & \text { if } k \leq m, \\ \left(\prod_{j=1}^{m} j^{n_{m}-n_{j}}\right)\left(\prod_{j=m+1}^{k} \frac{1}{j^{n_{j}-n_{m}}}\right) & \text { if } k>m .\end{cases}
$$

This implies (2.1) and (2.2). Hence Proposition 2.5 yields a weight $v$ with $r_{n_{m}}=s_{m}$. We obtain $\left|n_{m+1}-n_{m}\right|=m+1 \leq \min \left(n_{m}, n_{m+1}\right)$ and

$$
\left(\frac{s_{m}}{s_{m+1}}\right)^{n_{m}} \frac{v_{m}}{v_{m+1}}=(m+1)^{m+1} \text { and }\left(\frac{s_{m+1}}{s_{m}}\right)^{n_{m+1}} \frac{v_{m+1}}{v_{m}}=1 .
$$

This shows that $v$ satisfies $(\mathrm{C})$. Hence $H v \sim h v \sim H_{\infty}$.

3. Trigonometric polynomials. In the following let $[x]$ be the largest integer $\leq x$ for a given number $x \in \mathbb{R}$. We need

3.1. Lemma. Let $0<r<s$ and $m, n>0$.

(a) Then, for any trigonometric polynomial $f$ of degree $\leq n$, we have

$$
M_{\infty}(f, s) \leq\left(\frac{s}{r}\right)^{n} M_{\infty}(f, r) .
$$

(b) Let $g \in \operatorname{span}\left\{t^{|k|} \exp (i k \varphi):|k|>m\right\}$. Then

$$
M_{\infty}(g, r) \leq \frac{2(r / s)^{m}}{(r / s)^{2 m}+1} M_{\infty}(g, s) \leq 2\left(\frac{r}{s}\right)^{m} M_{\infty}(g, s) .
$$

Proof. (a) See [15, Lemma 3.1(i)].

(b) Put $p=[m]+1$. Let

$$
h(\exp (i \varphi))=\frac{1}{2}(\exp (i p \varphi)+\exp (-i p \varphi)) \sum_{k \in \mathbb{Z}}\left(\frac{r}{s}\right)^{|k|} \exp (i k \varphi) .
$$

Then $h$ is a Poisson kernel up to the factor $2^{-1}(\exp (i p \varphi)+\exp (-i p \varphi))$. Hence $(2 \pi)^{-1} \int_{0}^{2 \pi}|h(\exp (i \varphi))| d \varphi \leq 1$ and

$$
\begin{aligned}
h(\exp (i \varphi))= & \frac{1}{2} \sum_{j \geq p}\left(\left(\frac{r}{s}\right)^{j-p}+\left(\frac{r}{s}\right)^{j+p}\right) \exp (i j \varphi) \\
& +\frac{1}{2} \sum_{j \leq-p}\left(\left(\frac{r}{s}\right)^{p-j}+\left(\frac{r}{s}\right)^{-j-p}\right) \exp (i j \varphi)+\sum_{|j|<p} \alpha_{j} \exp (i j \varphi)
\end{aligned}
$$

for some $\alpha_{j}$. If $g=\sum_{|k|>m} \beta_{k} t^{|k|} \exp (i k \varphi)$ for some $\beta_{k}$ we obtain

$$
\frac{1}{2}\left(\left(\frac{r}{s}\right)^{p}+\left(\frac{s}{r}\right)^{p}\right) g(r \exp (i \varphi))=\frac{1}{2 \pi} \int_{0}^{2 \pi} h(\exp (i(\varphi-\psi))) g(s \exp (i \psi)) d \psi
$$


This implies, since $0<(r / s)^{p}<(r / s)^{m}<1$,

$$
\begin{aligned}
|g(r \exp (i \varphi))|= & 2\left(\left(\frac{r}{s}\right)^{p}+\left(\frac{s}{r}\right)^{p}\right)^{-1} \\
& \times(2 \pi)^{-1}\left|\int_{0}^{2 \pi} h(\exp (i(\varphi-\psi))) g(s \exp (i \psi)) d \psi\right| \\
\leq & \frac{2(r / s)^{p}}{(r / s)^{2 p}+1} M_{\infty}(g, s)(2 \pi)^{-1} \int_{0}^{2 \pi}|h(\exp (i(\varphi-\psi)))| d \psi \\
\leq & \frac{2(r / s)^{m}}{(r / s)^{2 m}+1} M_{\infty}(g, s) .
\end{aligned}
$$

Hence

$$
M_{\infty}(g, r) \leq \frac{2(r / s)^{m}}{(r / s)^{2 m}+1} M_{\infty}(g, s)
$$

Now, fix a weight $v:\left[0, a\left[\rightarrow \mathbb{R}_{+}\right.\right.$. As before, let $r_{m}$ be a maximum point of the function $r \mapsto r^{m} v(r), r>0$.

\subsection{Corollary.}

(a) Fix $m>0$ and consider $f \in \operatorname{span}\left\{r^{|k|} \exp (i k \varphi): k \in \mathbb{Z},|k| \leq m\right\}$, $g \in \operatorname{span}\left\{r^{|k|} \exp (i k \varphi): k \in \mathbb{Z},|k|>m\right\}$. Then

$$
\|f\|_{v} \leq \sup _{r \leq r_{m}} M_{\infty}(f, r) v(r) \quad \text { and } \quad\|g\|_{v} \leq 2 \sup _{r \geq r_{m}} M_{\infty}(g, r) v(r) .
$$

(b) Fix $0<m<n$ and put

$$
\alpha=\left(\frac{r_{m}}{r_{n}}\right)^{m} \frac{v\left(r_{m}\right)}{v\left(r_{n}\right)}, \quad \beta=\left(\frac{r_{n}}{r_{m}}\right)^{n} \frac{v\left(r_{n}\right)}{v\left(r_{m}\right)} .
$$

Then any $h \in \operatorname{span}\left\{r^{|k|} \exp (i k \varphi): k \in \mathbb{Z}, m<|k| \leq n\right\}$ satisfies

$$
\|h\|_{v} \leq 2 \alpha M_{\infty}\left(h, r_{n}\right) v\left(r_{n}\right) \text { and }\|h\|_{v} \leq 2 \beta M_{\infty}\left(h, r_{m}\right) v\left(r_{m}\right) .
$$

Proof. (a) If $r>r_{m}$ then we obtain, by Lemma 3.1,

$$
M_{\infty}(f, r) v(r) \leq\left(\frac{r}{r_{m}}\right)^{m} \frac{v(r)}{v\left(r_{m}\right)} M_{\infty}\left(f, r_{m}\right) v\left(r_{m}\right) \leq M_{\infty}\left(f, r_{m}\right) v\left(r_{m}\right) .
$$

If $0<r<r_{m}$ Lemma 3.1 implies

$$
M_{\infty}(g, r) v(r) \leq 2\left(\frac{r}{r_{m}}\right)^{m} \frac{v(r)}{v\left(r_{m}\right)} M_{\infty}\left(g, r_{m}\right) v\left(r_{m}\right) \leq 2 M_{\infty}\left(g, r_{m}\right) v\left(r_{m}\right) .
$$

This yields (a).

(b) According to (a) we have

$$
\begin{aligned}
\|h\|_{v} & \leq \sup _{r \leq r_{n}} M_{\infty}(h, r) v(r) \leq 2 \sup _{r \leq r_{n}}\left(\frac{r}{r_{n}}\right)^{m} \frac{v(r)}{v\left(r_{n}\right)} M_{\infty}\left(h, r_{n}\right) v\left(r_{n}\right) \\
& \leq 2 \alpha M_{\infty}\left(h, r_{n}\right) v\left(r_{n}\right)
\end{aligned}
$$


and

$$
\begin{aligned}
\|h\|_{v} & \leq 2 \sup _{r \geq r_{m}} M_{\infty}(h, r) v(r) \leq 2 \sup _{r \geq r_{m}}\left(\frac{r}{r_{m}}\right)^{n} \frac{v(r)}{v\left(r_{m}\right)} M_{\infty}\left(h, r_{m}\right) v\left(r_{m}\right) \\
& \leq 2 \beta M_{\infty}\left(h, r_{m}\right) v\left(r_{m}\right) .
\end{aligned}
$$

We want to study special operators on $h v$. Note that any linear operator $T: h v \rightarrow h v$ is bounded provided that $T$, restricted to the trigonometric polynomials, is bounded with respect to $M_{\infty}(\cdot, 1)$. Let $\|T\|_{v}$ be the operator norm with respect to $\|\cdot\|_{v}$ and $\|T\|_{\infty}$ the operator norm with respect to $M_{\infty}(\cdot, 1)$. We always have $\|T\|_{v} \leq\|T\|_{\infty}$. Indeed, put $z=r \exp (i \varphi)$ and $f=\sum_{k} \alpha_{k} r^{|k|} \exp (i k \varphi)$. Then

$$
\begin{aligned}
|(T f)(z)| v(|z|) & =\left|T\left(\sum_{k} \alpha_{k} r^{|k|} \exp (i k \varphi)\right)\right| v(r) \\
& \leq\|T\|_{\infty} \sup _{\varphi}\left|\sum_{k} \alpha_{k} r^{|k|} \exp (i k \varphi)\right| v(r) \leq\|T\|_{\infty}\|f\|_{v} .
\end{aligned}
$$

Hence $\|T f\|_{v} \leq\|T\|_{\infty}\|f\|_{v}$.

Sometimes $T$ is bounded with respect to $\|\cdot\|_{v}$ but unbounded with respect to $M_{\infty}(\cdot, 1)$ (see below).

Now fix $0<m<n$ (not necessarily integers) and consider the trigonometric polynomial $f=\sum_{k \in \mathbb{Z}} \alpha_{k} r^{|k|} \exp (i k \varphi)$. We define the operator $V_{n, m}$ by

$$
V_{n, m} f=\sum_{|k| \leq m} \alpha_{k} r^{|k|} \exp (i k \varphi)+\sum_{m<|k| \leq n} \frac{[n]-|k|}{[n]-[m]} \alpha_{k} r^{|k|} \exp (i k \varphi)
$$

Moreover, we consider the Riesz projection

$$
R f=\sum_{k \geq 0} \alpha_{k} r^{|k|} \exp (i k \varphi)
$$

3.3. LEMma. We have

(a) $\left\|V_{n, m}\right\|_{\infty} \leq \frac{[n]+[m]}{[n]-[m]}$

(b) $M_{\infty}(R h, r) \leq\left(1+\frac{[n]-[m]}{[m]}\right) M_{\infty}(h, r)$

for any $r>0$ and $h \in \operatorname{span}\left\{r^{|k|} \exp (i k \varphi): k \in \mathbb{Z}, m<|k| \leq n\right\}$,

(c) $\left\|V_{n_{4}, n_{3}}-V_{n_{2}, n_{1}}\right\|_{\infty} \leq 4 \frac{\left[n_{4}\right]-\left[n_{1}\right]}{\left[n_{2}\right]-\left[n_{1}\right]}\left(3+4 \frac{\left[n_{4}\right]-\left[n_{1}\right]}{\left[n_{4}\right]-\left[n_{3}\right]}\right)$

if $0<n_{1}<n_{2}<n_{3}<n_{4}$,

(d) $\left\|V_{n_{4}, n_{3}}-V_{n_{2}, n_{1}}\right\|_{\infty} \leq 2\left(\left[n_{4}\right]-\left[n_{1}\right]\right)$, $\left\|R\left(V_{n_{4}, n_{3}}-V_{n_{2}, n_{1}}\right)\right\|_{\infty} \leq\left[n_{4}\right]-\left[n_{1}\right]$ if $0<n_{1}<n_{2}<n_{3}<n_{4}$. 
Proof. (a) By definition we have $V_{n, m}=V_{[n],[m]}$. Fix $p \in \mathbb{Z}_{+}$. Then

$$
V_{p, 0} f=\sum_{|k| \leq p} \frac{p-|k|}{p} \alpha_{k} r^{|k|} \exp (i k \varphi) .
$$

It is well known ([11]) that $\left\|V_{p, 0}\right\|_{\infty}=1$. Since

$$
V_{n, m}=\frac{[n] V_{[n], 0}-[m] V_{[m], 0}}{[n]-[m]}
$$

we obtain (a).

(b) Let $m$ and $n$ be integers. Fix $k \in \mathbb{Z}$ and put, for the trigonometric polynomial $f,\left(S_{k} f\right)(r \exp (i \varphi))=\exp (i k \varphi) f(r \exp (i \varphi))$. If $h$ is as indicated in (b) we obtain $R h=S_{n} V_{n+m, n-m} S_{-n} h$ (compare the Fourier coefficients on both sides). We conclude that $M_{\infty}(R h, r) \leq 2 n(2 m)^{-1} M_{\infty}(h, r)$. From this the result follows.

(c) Retain the notation $S_{k}$ of (b). Let $0 \leq n_{1}<n_{2}<n_{3}<n_{4}$ be integers. Put $(U f)(z)=f(\bar{z})$ for any trigonometric polynomial $f$. Set $T=$ $V_{n_{4}+n_{2}-2 n_{1}, n_{3}+n_{2}-2 n_{1}}-V_{2\left(n_{2}-n_{1}\right), n_{2}-n_{1}}$. Then

$$
V_{n_{4}, n_{3}}-V_{n_{2}, n_{1}}=U S_{2 n_{1}-n_{2}} R T S_{-\left(2 n_{1}-n_{2}\right)} U+S_{2 n_{1}-n_{2}} R T S_{-\left(2 n_{1}-n_{2}\right)} .
$$

Hence (a) and (b) imply

$$
\begin{aligned}
\left\|V_{n_{4}, n_{3}}-V_{n_{2}, n_{1}}\right\|_{\infty} & \leq 2 \frac{n_{4}+n_{2}-2 n_{1}}{n_{2}-n_{1}}\left(3+\frac{n_{4}+n_{3}+2 n_{2}-4 n_{1}}{n_{4}-n_{3}}\right) \\
& \leq 4 \frac{n_{4}-n_{1}}{n_{2}-n_{1}}\left(3+4 \frac{n_{4}-n_{1}}{n_{4}-n_{3}}\right) .
\end{aligned}
$$
with

(d) Put $f=\sum_{k} \alpha_{k} \exp (i k \varphi)$. Then, by definition, there are $\varrho_{k} \in[0,1]$

$$
\begin{aligned}
\left(V_{n_{4}, n_{3}}-V_{n_{2}, n_{1}}\right) f & =\sum_{n_{1}<|k| \leq n_{4}} \alpha_{k} \varrho_{k} \exp (i k \varphi), \\
R\left(V_{n_{4}, n_{3}}-V_{n_{2}, n_{1}}\right) f & =\sum_{n_{1}<k \leq n_{4}} \alpha_{k} \varrho_{k} \exp (i k \varphi) .
\end{aligned}
$$

Since $\left|\alpha_{k}\right| \leq\|f\|_{\infty}$ for all $k,(\mathrm{~d})$ follows.

3.4. Proposition. Suppose that, for some $n, m>0$,

$$
\alpha:=\left(\frac{r_{n}}{r_{m}}\right)^{n} \frac{v\left(r_{n}\right)}{v\left(r_{m}\right)}>2 .
$$

(a) Then there is $\beta(\alpha)>0$ such that $\|f\|_{v} \leq \beta(\alpha)\|f+g\|_{v}$ whenever $f \in \operatorname{span}\left\{r^{|k|} \exp (i k \varphi): k \in \mathbb{Z},|k| \leq \min (m, n)\right\}$ and $g \in$ $\operatorname{span}\left\{r^{|k|} \exp (i k \varphi): k \in \mathbb{Z},|k|>\max (m, n)\right\} ;$ moreover, $\lim \sup _{\alpha \rightarrow \infty} \beta(\alpha)<\infty$. 
(b) There is a constant $\gamma(\alpha)>0$ such that $V:=V_{\max (m, n), \min (m, n)}$ : $h v \rightarrow$ hv satisfies $\|V\|_{v} \leq \gamma(\alpha)$; moreover, $\lim \sup _{\alpha \rightarrow \infty} \gamma(\alpha)<\infty$.

Proof. (a) First consider the case $m<n$. By Lemma 3.1 and Corollary 3.2 , we have

$$
\begin{aligned}
\|f+g\|_{v} & \geq \sup _{r \leq r_{m}} M_{\infty}(f+g, r) v(r) \\
& \geq \sup _{r \leq r_{m}}\left(M_{\infty}(f, r) v(r)-M_{\infty}(g, r) v(r)\right) \\
& \geq\|f\|_{v}-2\left(\frac{r_{m}}{r_{n}}\right)^{n} \frac{v\left(r_{m}\right)}{v\left(r_{n}\right)}\left(\sup _{r \leq r_{m}}\left(\frac{r}{r_{m}}\right)^{n} \frac{v(r)}{v\left(r_{m}\right)}\right) M_{\infty}\left(g, r_{n}\right) v\left(r_{n}\right) \\
& \geq\|f\|_{v}-\frac{2}{\alpha} \sup _{r \leq r_{m}}\left(\frac{r}{r_{m}}\right)^{m} \frac{v(r)}{v\left(r_{m}\right)}\|g\|_{v} \\
& \geq\|f\|_{v}-\frac{2}{\alpha}\|g\|_{v} \geq\|f\|_{v}-\frac{2}{\alpha}\|f+g\|_{v}-\frac{2}{\alpha}\|f\|_{v} .
\end{aligned}
$$

Hence $\|f\|_{v} \leq(1-2 / \alpha)^{-1}(1+2 / \alpha)\|f+g\|_{v}$.

For $n<m$ we have, by Lemma 3.1,

$$
\begin{aligned}
\|f+g\|_{v} & \geq \sup _{r \geq r_{m}} M_{\infty}(f+g, r) v(r) \\
& \geq \frac{1}{2}\|g\|_{v}-\left(\sup _{r \geq r_{m}}\left(\frac{r}{r_{m}}\right)^{n} \frac{v(r)}{v\left(r_{m}\right)}\right) M_{\infty}\left(f, r_{n}\right) v\left(r_{n}\right)\left(\frac{r_{m}}{r_{n}}\right)^{n} \frac{v\left(r_{m}\right)}{v\left(r_{n}\right)} \\
& \geq \frac{1}{2}\left(\|g\|_{v}-\frac{2}{\alpha} \sup _{r \geq r_{m}}\left(\frac{r}{r_{m}}\right)^{m} \frac{v(r)}{v\left(r_{m}\right)}\|f\|_{v}\right) \\
& =\frac{1}{2}\left(\|f\|_{v}-\|f+g\|_{v}-\frac{2}{\alpha}\|f\|_{v}\right)
\end{aligned}
$$

We obtain $\|f\|_{v} \leq(3 /(1-2 / \alpha))\|f+g\|_{v}$.

(b) Assume without loss of generality that $m<n$. Fix $h \in h v$, say $h=\sum_{k} \alpha_{k} r^{|k|} \exp (i k \varphi)$.

First consider the case $[m]=[n]$. Then, by definition, $V h=\sum_{|k| \leq m} \alpha_{k} r^{|k|}$ $\exp (i k \varphi)$. In view of (a) this means that $V$ is bounded by $\beta(\alpha)$.

Now assume $[n]-[m] \geq 1$. It suffices to assume $[n] \leq 2[m]$ (otherwise Proposition 3.4 follows from Lemma 3.3). Put $T=V_{2[n]-[m],[n]}-V_{[m], 2[m]-[n]}$. Lemma 3.3(a), (c) implies that $T$ is uniformly bounded. The definition of $T$ yields moreover $T\left(r^{|k|} \exp (i k \varphi)\right)=r^{|k|} \exp (i k \varphi)$ whenever $[m] \leq|k| \leq[n]$. Since $V=V_{[n],[m]}$ we obtain

$$
V T h=\left(V_{[n],[m]}-V_{[m], 2[m]-[n]}\right) h .
$$


Lemma 3.3(c) implies $\|V T h\|_{v} \leq 88\|h\|_{v}$. Now put

$$
P h=\sum_{|k|<m} \alpha_{k} r^{|k|} \exp (i k \varphi), \quad Q h=\sum_{|k|>n} \alpha_{k} r^{|k|} \exp (i k \varphi)
$$

and $f=P($ id $-T) h, g=Q($ id $-T) h$. We obtain $T h+f+g=h$.

(a) and the definitions of $V$ and $g$ imply

$$
\begin{aligned}
\|V h\|_{v} & =\|f+V T h\|_{v} \leq\|f\|_{v}+88\|h\|_{v} \leq \beta(\alpha)\|f+g\|_{v}+88\|h\|_{v} \\
& \leq \beta(\alpha)\|f+g+T h\|_{v}+\beta(\alpha)\|T h\|_{v}+88\|h\|_{v} \\
& \leq\left(\beta(\alpha)\left(1+\|T\|_{v}\right)+88\right)\|h\|_{v} .
\end{aligned}
$$

4. Conditions (B) and $\neg(\mathrm{B})$. Let $v:\left[0, a\left[\rightarrow \mathbb{R}_{+}\right.\right.$be a weight. First we prove

4.1. Proposition. Let $v$ satisfy $(\mathrm{B})$ and let $c>0$ be the corresponding constant in (B). Fix $c<m<n<p$ and $b, d>1$ such that $b \leq \alpha, \beta, \gamma, \delta \leq d$ where

$$
\begin{array}{ll}
\alpha=\left(\frac{r_{m}}{r_{n}}\right)^{m} \frac{v\left(r_{m}\right)}{v\left(r_{n}\right)}, & \beta=\left(\frac{r_{n}}{r_{m}}\right)^{n} \frac{v\left(r_{n}\right)}{v\left(r_{m}\right)}, \\
\gamma=\left(\frac{r_{n}}{r_{p}}\right)^{n} \frac{v\left(r_{n}\right)}{v\left(r_{p}\right)}, & \delta=\left(\frac{r_{p}}{r_{n}}\right)^{p} \frac{v\left(r_{p}\right)}{v\left(r_{n}\right)} .
\end{array}
$$

Then there are constants $d^{\prime}>1$ and $\kappa, \eta>0$ depending only on $b$ and $d$ but not on $m, n$ or $p$ such that either $p-m \leq c$ or

$$
\eta \leq \frac{p-n}{n-m} \leq \kappa \quad \text { and } \quad \max \left(\left(\frac{r_{m}}{r_{p}}\right)^{m} \frac{v\left(r_{m}\right)}{v\left(r_{p}\right)},\left(\frac{r_{p}}{r_{m}}\right)^{p} \frac{v\left(r_{p}\right)}{v\left(r_{m}\right)}\right) \leq d^{\prime} .
$$

Proof. Our assumptions imply

$$
\frac{r_{m}}{r_{n}} \leq\left(\frac{1}{b}\right)^{\frac{2}{n-m}} \text { and } \quad \frac{r_{n}}{r_{p}} \leq\left(\frac{1}{b}\right)^{\frac{2}{p-n}}
$$

Assume $p-m>c$.

If $n-m \leq p-n$ we have

$$
\left(\frac{r_{m}}{r_{p}}\right)^{m} \frac{v\left(r_{m}\right)}{v\left(r_{p}\right)}=\alpha \gamma\left(\frac{r_{p}}{r_{n}}\right)^{n-m} \leq \alpha \gamma\left(\frac{r_{p}}{r_{n}}\right)^{p-n} \leq \alpha \gamma^{2} \delta \leq d^{4} .
$$

(B) provides us with a constant $b^{\prime}=b^{\prime}\left(d^{4}\right)>1$ such that $\left(r_{p} / r_{m}\right)^{p} v\left(r_{p}\right) / v\left(r_{m}\right)$ $\leq b^{\prime}$. In this case we have

$$
\left(\frac{1}{b^{\prime} d^{4}}\right)^{\frac{1}{p-m}} \leq \frac{r_{m}}{r_{p}} \leq\left(\frac{1}{b}\right)^{\frac{2}{n-m}+\frac{2}{p-n}}
$$

which implies

$$
2(\log b)\left(\frac{1}{n-m}+\frac{1}{p-n}\right) \leq \frac{\log \left(b^{\prime} d^{4}\right)}{p-m} .
$$


Since $p-m=(p-n)+(n-m)$ we deduce

$$
1 \leq \max \left(\frac{p-n}{n-m}, \frac{n-m}{p-n}\right) \leq \frac{\log \left(b^{\prime} d^{4}\right)}{2 \log b} .
$$

If $p-n<n-m$ we have

$$
\left(\frac{r_{p}}{r_{m}}\right)^{p} \frac{v\left(r_{p}\right)}{v\left(r_{m}\right)}=\delta \beta\left(\frac{r_{n}}{r_{m}}\right)^{p-n} \leq \delta \beta\left(\frac{r_{n}}{r_{m}}\right)^{n-m} \leq \delta \beta^{2} \alpha \leq d^{4}
$$

and we proceed exactly as before. Put $d^{\prime}=\max \left(d^{4}, b^{\prime}\right)$.

In order to discuss some consequences of 4.1 we need two technical lemmas.

4.2. Lemma. Let $b_{1}, b_{2}>1$ and $m, n>0$ be such that

$$
\left(\frac{r_{n}}{r_{m}}\right)^{n} \frac{v\left(r_{n}\right)}{v\left(r_{m}\right)} \geq b_{2} \quad \text { and } \quad\left(\frac{r_{m}}{r_{n}}\right)^{m} \frac{v\left(r_{m}\right)}{v\left(r_{n}\right)} \leq b_{1} .
$$

Then for any $N \in \mathbb{Z}_{+}$and $p=n 2^{-N}+\left(1-2^{-N}\right) m$, we have

$$
\left(\frac{r_{p}}{r_{m}}\right)^{p} \frac{v\left(r_{p}\right)}{v\left(r_{m}\right)} \geq b_{2}^{1 / 2^{N}} b_{1}^{-1+1 / 2^{N}}, \quad\left(\frac{r_{m}}{r_{p}}\right)^{m} \frac{v\left(r_{m}\right)}{v\left(r_{p}\right)} \leq b_{1}
$$

and $|p-m| 2^{N}=|n-m|$.

Proof. First, for $n_{1}=(m+n) / 2$ we easily obtain

$$
\left(\frac{r_{n}}{r_{m}}\right)^{n_{1}} \frac{v\left(r_{n}\right)}{v\left(r_{m}\right)} \geq \sqrt{\frac{b_{2}}{b_{1}}} .
$$

Hence

$$
\left(\frac{r_{n_{1}}}{r_{m}}\right)^{n_{1}} \frac{v\left(r_{n_{1}}\right)}{v\left(r_{m}\right)}=\left(\frac{r_{n}}{r_{m}}\right)^{n_{1}} \frac{v\left(r_{n}\right)}{v\left(r_{m}\right)}\left(\frac{r_{n_{1}}}{r_{n}}\right)^{n_{1}} \frac{v\left(r_{n_{1}}\right)}{v\left(r_{n}\right)} \geq \sqrt{\frac{b_{2}}{b_{1}}} .
$$

Since $\left(r_{n} / r_{n_{1}}\right)^{m} \leq\left(r_{n} / r_{n_{1}}\right)^{n_{1}}$ for $m \leq n_{1} \leq n$ as well as for $n \leq n_{1} \leq m$ we also obtain

$$
\left(\frac{r_{m}}{r_{n_{1}}}\right)^{m} \frac{v\left(r_{m}\right)}{v\left(r_{n_{1}}\right)}=\left(\frac{r_{m}}{r_{n}}\right)^{m} \frac{v\left(r_{m}\right)}{v\left(r_{n}\right)}\left(\frac{r_{n}}{r_{n_{1}}}\right)^{m} \frac{v\left(r_{n}\right)}{v\left(r_{n_{1}}\right)} \leq b_{1}\left(\frac{r_{n}}{r_{n_{1}}}\right)^{n_{1}} \frac{v\left(r_{n}\right)}{v\left(r_{n_{1}}\right)} \leq b_{1} .
$$

In the next step we repeat the procedure with $n_{1}$ instead of $n$ and $\sqrt{b_{2} / b_{1}}$ instead of $b_{2}$. This yields $n_{2}=\left(n_{1}+m\right) / 2$ and

$$
\left(\frac{r_{n_{2}}}{r_{m}}\right)^{n_{2}} \frac{v\left(r_{n_{2}}\right)}{v\left(r_{m}\right)} \geq b_{2}^{1 / 4} b_{1}^{-1 / 2-1 / 4}, \quad\left(\frac{r_{m}}{r_{n_{2}}}\right)^{m} \frac{v\left(r_{m}\right)}{v\left(r_{n_{2}}\right)} \leq b_{1} .
$$

Continuation proves Lemma 4.2 .

4.3. Lemma. Fix $M, q \in \mathbb{Z}_{+}$and put

$$
P_{q, M}(f)=\sum_{j} \alpha_{q+j M} r^{|q+j M|} \exp (i(q+j M) \varphi)
$$

for any trigonometric polynomial $f=\sum_{k} \alpha_{k} r^{|k|} \exp (i k \varphi)$. Then $\left\|P_{q, M}\right\|_{\infty}=1$. 
Proof. We obtain

$$
\begin{gathered}
\frac{1}{M} \sum_{l=0}^{M-1} \exp \left(-i \frac{2 \pi}{M} l q\right) f\left(\exp \left(i \frac{2 \pi}{M} l\right) \cdot r \exp (i \varphi)\right) \\
=\frac{1}{M} \sum_{k} \alpha_{k}\left(\sum_{l=0}^{M-1} \exp \left(i \frac{2 \pi}{M} l(k-q)\right)\right) r^{|k|} \exp (i k \varphi) \\
=\sum_{j} \alpha_{q+j M} r^{|q+j M|} \exp (i(q+j M) \varphi) .
\end{gathered}
$$

This implies that $P_{q, M}$ has norm one.

Again let $H_{n}=\operatorname{span}\left\{1, z, \ldots, z^{n}\right\}$ be endowed with $M_{\infty}(\cdot, 1)$. Now we are ready to prove

4.4. Proposition. Assume $\neg(\mathrm{B})$. Fix $M, N \in \mathbb{Z}_{+}$. Then there is a subspace $A \subset \operatorname{span}\left\{z^{k}: k \geq M\right\} \subset(H v)_{0}$ and a projection $Q: H v \rightarrow A$ such that $\|Q\|_{v}$ and the Banach-Mazur distance $d\left(A, H_{N}\right)$ do not depend on $M$ or $N$. If, in addition, $v$ satisfies (C) then $Q$ is defined and uniformly bounded on all of $h v$.

Proof. $\neg(\mathrm{B})$ yields the existence of $b>1$ and $m, n \geq \max (N, M)$, with

$$
\left(\frac{r_{m}}{r_{n}}\right)^{m} \frac{v\left(r_{m}\right)}{v\left(r_{n}\right)} \leq b, \quad\left(\frac{r_{n}}{r_{m}}\right)^{n} \frac{v\left(r_{n}\right)}{v\left(r_{m}\right)} \geq b^{2^{N+1}}
$$

and $|m-n| \geq N 2^{N}$. We may even assume that

$$
b>2 .
$$

According to Lemma 4.2 we find $p$ between $m$ and $n$ with

$$
\begin{gathered}
|n-m|=2^{N}|p-m| \\
\left(\frac{r_{p}}{r_{m}}\right)^{p} \frac{v\left(r_{p}\right)}{v\left(r_{m}\right)} \geq b \text { and }\left(\frac{r_{m}}{r_{p}}\right)^{m} \frac{v\left(r_{m}\right)}{v\left(r_{p}\right)} \leq b
\end{gathered}
$$

In particular we have $|n-p| \geq\left(2^{N}-1\right)|p-m|$. Corollary 3.2 implies

$$
\|f\|_{v} \leq 2 b M_{\infty}\left(f, r_{n}\right) v\left(r_{n}\right)
$$

whenever $f \in \operatorname{span}\left\{r^{|k|} \exp (i k \varphi):|k|\right.$ between $n$ and $\left.m\right\}$.

CASE $m<p<n$. Then, in view of Proposition 3.4(b), $\left\|V_{p, m}\right\|_{v}$ does not depend on $m$ or $p$ (see (4.1) and (4.3)). We may assume without loss of generality from now on that $m$ and $p$ are integers. Otherwise we take $[m]$ and $[p]$ instead.

Put $Q_{1}=P_{m, p-m}\left(\mathrm{id}-V_{p, m}\right)\left(P_{m, p-m}\right.$ as in Lemma 4.3). Then, for $k \geq 0$,

$$
Q_{1}\left(z^{k}\right)= \begin{cases}z^{k} & \text { if } k=p+j(p-m) \text { for some integer } j \geq 0 \\ 0 & \text { else. }\end{cases}
$$


Define $T_{1}: H_{N} \rightarrow(H v)_{0}$ by

$$
T_{1} z^{j}=\frac{z^{p+j(p-m)}}{r_{n}^{p+j(p-m)} v\left(r_{n}\right)}, \quad j=0,1, \ldots, N .
$$

Since $p+N(p-m)=m+(N+1)(p-m) \leq n$ (see (4.2)) we obtain $\left\|T_{1}\right\| \leq 2 b$ $(\operatorname{see}(4.4))$.

Define $\widetilde{S}_{1}: H v \rightarrow L_{\infty}(\partial D)$ by

$$
\left(\widetilde{S}_{1} f\right)(z)=\left(Q_{1} f\right)\left(r_{n} z^{1 /(p-m)}\right) \cdot \bar{z}^{p /(p-m)} v\left(r_{n}\right), \quad f \in H v
$$

which implies

$$
\widetilde{S}_{1} z^{k}= \begin{cases}r_{n}^{k} z^{j} v\left(r_{n}\right) & \text { if } k=p+j(p-m) \text { for some integer } j \geq 0 \\ 0 & \text { else }\end{cases}
$$

(see (4.5)). Finally, put

$$
S_{1}=V_{N, 0} \widetilde{S}_{1}
$$

Then (4.3), Proposition 3.4 and the definition of $Q_{1}$ imply that $\left\|S_{1}\right\| \leq \gamma(b)$ for some $\gamma(b)>0$ which does not depend on $m, n$ or $p$. (Recall that $N \leq n$.) Moreover, (4.6) and (4.7) show that $S_{1} T_{1}=\left.V_{N, 0}\right|_{H_{N}}$.

CAsE $n<p<m$. Here $\left\|V_{m, p}\right\|_{v}$ does not depend on $m$ or $p$. As before, we may assume from now on that $m$ and $p$ are integers.

Put $Q_{1}=P_{m, m-p} V_{m, p}$. Then

$$
Q_{1} z^{k}= \begin{cases}z^{k} & \text { if } k=p-j(m-p) \text { for some integer } j \geq 0 \\ 0 & \text { else. }\end{cases}
$$

Define $\widetilde{S}_{1}: H v \rightarrow L_{\infty}(\partial D)$ by

$$
\left(\widetilde{S}_{1} f\right)(z)=\left(Q_{1} f\right)\left(r_{n} \bar{z}^{1 /(m-p)}\right) \cdot z^{p /(m-p)} v\left(r_{n}\right), \quad f \in H v
$$

so that

$$
\widetilde{S}_{1} z^{k}= \begin{cases}r_{n}^{k} z^{j} v\left(r_{n}\right) & \text { if } k=p-j(m-p) \text { for some integer } j \geq 0 \\ 0 & \text { else. }\end{cases}
$$

Then put $S_{1}=V_{N, 0} \widetilde{S}_{1}$. Finally, define $T_{1}: H_{N} \rightarrow(H v)_{0}$ by

$$
T_{1} z^{j}=\frac{z^{p-j(m-p)}}{r_{n}^{p-j(m-p)} v\left(r_{n}\right)}, \quad j=0,1, \ldots, N .
$$

As before we obtain $S_{1} T_{1}=\left.V_{N, 0}\right|_{H_{N}}$ and $\left\|S_{1}\right\| \leq \gamma(b),\left\|T_{1}\right\| \leq 2 b$.

In both cases we have $S_{1} z^{k}=0$ if $k$ is not between $n$ and $m$ (see (4.2), (4.7), (4.8) and take into account that $\min (m, n)+N|m-p| \leq \max (m, n))$. Now, fix $M_{1}>\max (M, m, n)$. Repeat the same procedure with $M_{1}$ instead of $M$ to find $m^{\prime} \geq M_{1}, n^{\prime} \geq M_{1}$ and linear operators $T_{2}: H_{N} \rightarrow(H v)_{0}$ and $S_{2}: H v \rightarrow H_{N}$ such that $\left\|S_{2}\right\| \leq \gamma(b),\left\|T_{2}\right\| \leq 2 b, S_{2} T_{2}=\left.V_{N, 0}\right|_{H_{N}}$, and 
$S_{2} z^{k}=0$ if $k$ is not between $m^{\prime}$ and $n^{\prime}$. In particular

$$
S_{2} T_{1}=0 \quad \text { and } \quad S_{1} T_{2}=0 .
$$

For a complex function $f$ put $(W f)(z)=f(\bar{z})$. Finally, define $V:\left(H_{N} \oplus\right.$ $\left.H_{N}\right)_{\infty} \rightarrow H v$ by $V(f, g)=T_{1} f+T_{2} g$ and $U: H v \rightarrow\left(H_{N} \oplus H_{N}\right)_{\infty}$ by

$$
U f=\left(S_{1} f+z^{N} W S_{2} f, S_{2} f+z^{N} W S_{1} f\right) .
$$

Then $\|U\| \leq 2 \gamma(b)$ and $\|V\| \leq 4 b$. It is easily seen that $U H v=\operatorname{span}\left\{\left(z^{j}, z^{N-j}\right)\right.$ : $j=0,1, \ldots, N\}$, which is isometrically isomorphic to $H_{N}$. Moreover, by (4.9),

$$
\begin{aligned}
U V\left(z^{j}, z^{N-j}\right) & =U\left(T_{1} z^{j}+T_{2} z^{N-j}\right) \\
& =\left(V_{N, 0} z^{j}+z^{N} V_{N, 0} \bar{z}^{N-j}, V_{N, 0} z^{N-j}+z^{N} V_{N, 0} \bar{z}^{j}\right) \\
& =\left(z^{j}, z^{N-j}\right) .
\end{aligned}
$$

This implies that $Q=V U: H v \rightarrow H v$ is a projection and $d\left(Q H v, H_{N}\right)$ and $\|Q\|_{v}$ depend only on $b$. The construction of $Q$ and $U$ furthermore shows that $Q z^{k}=0$ if $k$ is neither between $m$ and $n$ nor between $m^{\prime}$ and $n^{\prime}$.

Now assume that, moreover, (C) holds. Then we can choose $m, m^{\prime}$ and $n, n^{\prime}$ such that, in addition,

$$
\begin{aligned}
& \min \left(m^{\prime}, n^{\prime}\right) \geq 3 \max (m, n), \quad \min (m, n) \geq d|n-m|, \\
& \min \left(m^{\prime}, n^{\prime}\right) \geq d\left|n^{\prime}-m^{\prime}\right|
\end{aligned}
$$

for some $d>0$, say $m<n<m^{\prime}<n^{\prime}$. Again we may assume that $m, m^{\prime}$, $n, n^{\prime}$ are integers (otherwise take $[m],\left[m^{\prime}\right],[n],\left[n^{\prime}\right]$ instead). Using (C) we can assume that

$$
\frac{d}{2}(n-m)>1 \text { and } \quad \frac{d}{2}\left(n^{\prime}-m^{\prime}\right)>1 .
$$

Define $W: h v \rightarrow H v$ by

$$
W=R\left(V_{n+\frac{d}{2}(n-m), n}-V_{m, m-\frac{d}{2}(n-m)}\right)+R\left(V_{n^{\prime}+\frac{d}{2}\left(n^{\prime}-m^{\prime}\right), n^{\prime}}-V_{m^{\prime}, m^{\prime}-\frac{d}{2}\left(n^{\prime}-m^{\prime}\right)}\right)
$$

where $R$ is the Riesz projection. From (4.10) we infer that $n+2^{-1} d(n-m)<$ $m^{\prime}-2^{-1} d\left(n^{\prime}-m^{\prime}\right)$. Lemma 3.3(b), (c) provides us with a constant $\alpha>0$ such that

$$
\begin{aligned}
\|W\|_{v} & =\alpha\left(1+\frac{(1+d)(n-m)}{m-\frac{d}{2}(n-m)}+1+\frac{(1+d)\left(n^{\prime}-m^{\prime}\right)}{m^{\prime}-\frac{d}{2}\left(n^{\prime}-m^{\prime}\right)}\right) \\
& \leq \alpha\left(2+4 \frac{1+d}{d}\right) .
\end{aligned}
$$

The construction yields $W z^{j}=z^{j}$ if $m \leq j \leq n$ or $m^{\prime} \leq j \leq n^{\prime}$. Finally, define $\widehat{Q}: h v \rightarrow Q H v$ by $\widehat{Q}=Q W$.

We deduce 
4.5. Corollary. Under the assumptions of Proposition 4.4 the spaces $H v$ and hv each contain a complemented subspace isomorphic to $H_{\infty}$ while $(H v)_{0}$ and $(h v)_{0}$ each contain a complemented subspace isomorphic to $\left(\sum_{n} \oplus H_{n}\right)_{0}$.

Proof. Let $c$ be a constant such that $d\left(A, H_{N}\right) \leq c$ and $\|Q\| \leq c$ for $A$, $H_{N}, Q$ of Proposition 4.4. Observe that for every $\varepsilon, M>0$ there is $K>0$ such that if $f \in \operatorname{span}\left\{r^{|k|} \exp (i k \varphi):|k| \leq M\right\}$ and $g \in \operatorname{span}\left\{r^{|k|} \exp (i \varphi)\right.$ : $|k| \geq N\}$ with $N-M \geq K$, then

$$
(1-\varepsilon) \max \left(\|f\|_{v},\|g\|_{v}\right) \leq\|f+g\|_{v} \leq(1+\varepsilon) \max \left(\|f\|_{v},\|g\|_{v}\right) .
$$

This follows since $\lim _{r \rightarrow a} v(r)=0$.

Using Proposition 4.4, by induction, we find integers $0<M_{1}<M_{2}<\cdots$ (sufficiently far apart), subspaces $A_{k} \subset(H v)_{0}$ and projections $Q_{k}: H v \rightarrow$ $A_{k}$ (or $\left.Q_{k}: h v \rightarrow A_{k}\right)$ such that $d\left(A_{k}, H_{k}\right) \leq c,\left\|Q_{k}\right\| \leq c$ and, for $T_{k}=$ $V_{M_{4 k+3}, M_{4 k+2}}-V_{M_{4 k+1}, M_{4 k}}$,

$$
\frac{1}{2} \sup _{k}\left\|T_{k} f\right\|_{v} \leq\left\|\sum_{k} T_{k} f\right\|_{v} \leq 2 \sup _{k}\left\|T_{k} f\right\|_{v}
$$

for all $f \in h v$ and

$$
T_{k} h=h \quad \text { for all } h \in A_{k}, k=1,2, \ldots
$$

Put $Q=\sum_{k} Q_{k} T_{k}$. Then, in view of (4.12) and (4.13), $Q$ is a bounded projection from $(H v)_{0}$ (or $\left.(h v)_{0}\right)$ onto the closure of $\operatorname{span}\left(\bigcup_{k=1}^{\infty} A_{k}\right)$ in $(H v)_{0}$.

Moreover, if the $f_{k} \in A_{k}$ are such that $\sup _{k}\left\|f_{k}\right\|_{v}<\infty$ then, in view of (4.12) and Montel's theorem, $\sum_{k} f_{k}$ converges (uniformly on compact subsets) to a holomorphic function (called $\sum_{k} f_{k}$ again) with $\left\|\sum_{k} f_{k}\right\|_{v}$ $<\infty$. Hence $\sum_{k} f_{k} \in H v$. We conclude that $\left\{\sum_{k} f_{k}: f_{k} \in A_{k}, k=1,2, \ldots\right.$, $\left.\sup _{k}\left\|f_{k}\right\|_{v}<\infty\right\}$ is complemented in $H v$ (or $h v$ ). Finally, this space is isomorphic to $\left(\sum_{n} \oplus H_{n}\right)_{(\infty)} \sim H_{\infty}$.

5. Norms equivalent to $\|\cdot\|_{v}$. First we prove, for a given weight $v:\left[0, a\left[\rightarrow \mathbb{R}_{+}\right.\right.$,

5.1. Lemma. Fix $b>1$. Then there are numbers $0<m_{1}<m_{2}<\cdots$ such that

$$
\left(\frac{r_{m_{n+1}}}{r_{m_{n}}}\right)^{m_{n+1}} \frac{v\left(r_{m_{n+1}}\right)}{v\left(r_{m_{n}}\right)} \geq b \quad \text { and } \quad\left(\frac{r_{m_{n}}}{r_{m_{n+1}}}\right)^{m_{n}} \frac{v\left(r_{m_{n}}\right)}{v\left(r_{m_{n+1}}\right)} \geq b,
$$

and, for each $n$, one of these inequalities is an equality; moreover, $\lim _{n \rightarrow \infty} m_{n}$ $=\infty$.

Proof. Start with $m_{1}=1$. Then assume that we already have $m_{n}$ for some $n$. Use $\lim _{M \rightarrow \infty} r_{M}^{m_{n}} v\left(r_{M}\right)=0$ (by assumption on $v$ ) to find $M_{0}>m_{n}$ 
with

$$
\left(\frac{r_{m_{n}}}{r_{M}}\right)^{m_{n}} \frac{v\left(r_{m_{n}}\right)}{v\left(r_{M}\right)} \geq b \quad \text { for any } M \geq M_{0} .
$$

Fix $M \geq M_{0}$ with $r_{M}>r_{m_{n}}$ and use

$$
\lim _{N \rightarrow \infty}\left(\frac{r_{M}}{r_{m_{n}}}\right)^{N} \frac{v\left(r_{M}\right)}{v\left(r_{m_{n}}\right)}=\infty
$$

to find $N>M$ with

$$
\left(\frac{r_{M}}{r_{m_{n}}}\right)^{N} \frac{v\left(r_{M}\right)}{v\left(r_{m_{n}}\right)} \geq b
$$

Since $r_{N}^{N} v\left(r_{N}\right) \geq r_{M}^{N} v\left(r_{M}\right)$ by definition of $r_{N}$, this implies

$$
\left(\frac{r_{N}}{r_{m_{n}}}\right)^{N} \frac{v\left(r_{N}\right)}{v\left(r_{m_{n}}\right)} \geq b \text { and }\left(\frac{r_{m_{n}}}{r_{N}}\right)^{m_{n}} \frac{v\left(r_{m_{n}}\right)}{v\left(r_{N}\right)} \geq b .
$$

Now let $N$ be the smallest number $>m_{n}$ which satisfies the last two inequalities and put $m_{n+1}=N$ (which exists since $m \mapsto r_{m}^{m} v\left(r_{m}\right)$ is continuous). Then, in particular, one of the above inequalities is an equality.

Finally, if $\sup _{n} m_{n}<\infty$ we would obtain

$$
\begin{aligned}
b & \leq \lim _{n \rightarrow \infty}\left(\frac{r_{m_{n+1}}}{r_{m_{n}}}\right)^{m_{n+1}} \frac{v\left(r_{m_{n+1}}\right)}{v\left(r_{m_{n}}\right)} \\
& =\lim _{n \rightarrow \infty} r_{m_{n}}^{m_{n}-m_{n+1}} \frac{r_{m_{n+1}}^{m_{n+1}} v\left(r_{m_{n+1}}\right)}{r_{m_{n}}^{m_{n}} v\left(r_{m_{n}}\right)}=1
\end{aligned}
$$

by continuity, a contradiction.

In the following let $b, m_{n}$ be the numbers of Lemma 5.1.

5.2. Proposition. Assume that $b>2$. Then there are constants $c_{1}, c_{2}$ $>0$ such that, for any $f \in h v$ and $f_{n}=\left(V_{m_{n+1}, m_{n}}-V_{m_{n}, m_{n-1}}\right) f$, we have

$$
\begin{aligned}
c_{1} \sup _{n} \sup _{r_{m_{n-1}} \leq r \leq r_{m_{n+1}}} & M_{\infty}\left(f_{n}, r\right) v(r) \\
& \leq\|f\|_{v} \leq c_{2} \sup _{n} \sup _{r_{m_{n-1}} \leq r \leq r_{m_{n+1}}} M_{\infty}\left(f_{n}, r\right) v(r) .
\end{aligned}
$$

Proof. The left-hand inequality is clear since, according to Proposition 3.4 , the operators $V_{m_{n+1}, m_{n}}-V_{m_{n}, m_{n-1}}$ are uniformly bounded with respect to $\|\cdot\|_{v}$. It suffices to assume that $f$ is a trigonometric polynomial. We have $f=\sum_{k} f_{k}$ and $f_{k} \in \operatorname{span}\left\{r^{|j|} \exp (i j \varphi):\left[m_{k-1}\right]+1 \leq|j| \leq\left[m_{k+1}\right]\right\}$. Fix $n$ and $r$ such that $r_{m_{n-1}} \leq r \leq r_{m_{n}}$. Then we obtain, using Lemma 3.1,

$$
M_{\infty}(f, r) v(r) \leq \sum_{k} M_{\infty}\left(f_{k}, r\right) v(r)
$$




$$
\begin{aligned}
& \leq \sum_{k \leq n-2}\left(\frac{r}{r_{m_{k+1}}}\right)^{m_{k+1}} \frac{v(r)}{v\left(r_{m_{k+1}}\right)} M_{\infty}\left(f_{k}, r_{m_{k+1}}\right) v\left(r_{m_{k+1}}\right) \\
& \quad+\sum_{j=-1}^{1} M_{\infty}\left(f_{n+j}, r\right) v(r) \\
& \quad+2 \sum_{k \geq n+2}\left(\frac{r}{r_{m_{k-1}}}\right)^{m_{k-1}} \frac{v(r)}{v\left(r_{m_{k-1}}\right)} M_{\infty}\left(f_{k}, r_{m_{k-1}}\right) v\left(r_{m_{k-1}}\right) .
\end{aligned}
$$

We have

$$
\begin{gathered}
\left(\frac{r}{r_{m_{k+1}}}\right)^{m_{k+1}} \frac{v(r)}{v\left(r_{m_{k+1}}\right)} \leq\left(\frac{r_{m_{k+2}}}{r_{m_{k+1}}}\right)^{m_{k+1}} \frac{v\left(r_{m_{k+2}}\right)}{v\left(r_{m_{k+1}}\right)}\left(\frac{r_{m_{k+3}}}{r_{m_{k+2}}}\right)^{m_{k+2}} \frac{v\left(r_{m_{k+3}}\right)}{v\left(r_{m_{k+2}}\right)} \\
\ldots\left(\frac{r_{m_{n-1}}}{r_{m_{n-2}}}\right)^{m_{n-2}} \frac{v\left(r_{m_{n-1}}\right)}{v\left(r_{m_{n-2}}\right)}\left(\frac{r}{r_{m_{n-1}}}\right)^{m_{n-1}} \frac{v(r)}{v\left(r_{m_{n-1}}\right)} \leq\left(\frac{1}{b}\right)^{n-k-2}
\end{gathered}
$$

if $k \leq n-2$ and, similarly, if $k \geq n+2$,

$$
\begin{array}{r}
\left(\frac{r}{r_{m_{k-1}}}\right)^{m_{k-1}} \frac{v(r)}{v\left(r_{m_{k-1}}\right)} \leq\left(\frac{r}{r_{m_{n+1}}}\right)^{m_{n+1}} \frac{v(r)}{v\left(r_{m_{n+1}}\right)}\left(\frac{r_{m_{n+1}}}{r_{m_{n+2}}}\right)^{m_{n+2}} \frac{v\left(r_{m_{n+1}}\right)}{v\left(r_{m_{n+2}}\right)} \\
\cdots\left(\frac{r_{m_{k-2}}}{r_{m_{k-1}}}\right)^{m_{k-1}} \frac{v\left(r_{m_{k-2}}\right)}{v\left(r_{m_{k-1}}\right)} \leq\left(\frac{1}{b}\right)^{k-1-n} .
\end{array}
$$

Since $b>1$ we obtain

$$
M_{\infty}(f, r) v(r) \leq c_{2} \sup _{n} \sup _{r_{m_{n-1}} \leq r \leq r_{m_{n+1}}} M_{\infty}\left(f_{n}, r\right) v(r)
$$

for some constant $c_{2}$ which depends only on $b$.

Using Proposition 5.2 it might be possible to exactly describe all the weights $\widetilde{v}$ such that the differentiation operator Diff $: H v \rightarrow H \widetilde{v}$, where $\operatorname{Diff}(f)=f^{\prime}$, is bounded.

We want to strengthen Proposition 5.2. To this end fix $n$ and find $p_{n}, q_{n}$ with $m_{n-1}<p_{n}<m_{n}<q_{n}<m_{n+1}$ such that

$$
\left(\frac{r_{p_{n}}}{r_{m_{n}}}\right)^{p_{n}} \frac{v\left(r_{p_{n}}\right)}{v\left(r_{m_{n}}\right)}=\sqrt{b} \text { and }\left(\frac{r_{q_{n}}}{r_{m_{n}}}\right)^{q_{n}} \frac{v\left(r_{q_{n}}\right)}{v\left(r_{m_{n}}\right)}=\sqrt{b}
$$

(Again, use the continuity of $p \mapsto r_{p}^{p} v\left(r_{p}\right)$.)

5.3. Lemma. Assume that $b>4$. Then there are universal constants $d_{1}, d_{2}>0$ such that, for every $n$, there is $s_{n} \in\left\{r_{m_{n}}, r_{m_{n+1}}\right\}$ satisfying the following.

For every $f \in \operatorname{span}\left\{r^{|k|} \exp (i k \varphi): m_{n-1} \leq|k| \leq m_{n+1}\right\}$ and $u_{n}=$ $V_{m_{n}, p_{n}} f, v_{n}=\left(V_{q_{n}, m_{n}}-V_{m_{n}, p_{n}}\right) f, w_{n}=\left(\mathrm{id}-V_{q_{n}, m_{n}}\right) f$, we have

$$
\begin{aligned}
\left\|u_{n}\right\|_{v} & \leq d_{2} M_{\infty}\left(u_{n}, s_{n-1}\right) v\left(s_{n-1}\right) \\
\left\|v_{n}\right\|_{v} & \leq d_{2} M_{\infty}\left(v_{n}, r_{m_{n}}\right) v\left(r_{m_{n}}\right) \\
\left\|w_{n}\right\|_{v} & \leq d_{2} M_{\infty}\left(w_{n}, s_{n}\right) v\left(s_{n}\right)
\end{aligned}
$$


In particular,

$$
\begin{gathered}
d_{1} \max \left(M_{\infty}\left(u_{n}, s_{n-1}\right) v\left(s_{n-1}\right), M_{\infty}\left(v_{n}, r_{m_{n}}\right) v\left(r_{m_{n}}\right), M_{\infty}\left(w_{n}, s_{n}\right) v\left(s_{n}\right)\right) \leq\|f\|_{v} \\
\leq d_{2} \max \left(M_{\infty}\left(u_{n}, s_{n-1}\right) v\left(s_{n-1}\right), M_{\infty}\left(v_{n}, r_{m_{n}}\right) v\left(r_{m_{n}}\right), M_{\infty}\left(w_{n}, s_{n}\right) v\left(s_{n}\right)\right) .
\end{gathered}
$$

Proof. According to the choice of $p_{n}$ and $q_{n}$, in view of Proposition 3.4, the norms of the operators $V_{m_{n}, p_{n}}$ and $V_{q_{n}, m_{n}}$ depend only on $b$. We have

$$
\begin{aligned}
& u_{n} \in \operatorname{span}\left\{r^{|k|} \exp (i k \varphi): m_{n-1} \leq|k| \leq m_{n}\right\}, \\
& v_{n} \in \operatorname{span}\left\{r^{|k|} \exp (i k \varphi): p_{n} \leq|k| \leq q_{n}\right\}, \\
& w_{n} \in \operatorname{span}\left\{r^{|k|} \exp (i k \varphi): m_{n} \leq|k| \leq m_{n+1}\right\} .
\end{aligned}
$$

Fix $j$. If

$$
\left(\frac{r_{m_{j+1}}}{r_{m_{j}}}\right)^{m_{j+1}} \frac{v\left(r_{m_{j+1}}\right)}{v\left(r_{m_{j}}\right)}=b
$$

put $s_{j}=r_{m_{j}}$. If this is not the case then, in view of Lemma 5.1, we have

$$
\left(\frac{r_{m_{j}}}{r_{m_{j+1}}}\right)^{m_{j}} \frac{v\left(r_{m_{j}}\right)}{v\left(r_{m_{j+1}}\right)}=b
$$

Here put $s_{j}=r_{m_{j+1}}$. Using Corollary 3.2 we deduce

$$
\begin{aligned}
\left\|u_{n}\right\|_{v} & \leq 2 b M_{\infty}\left(u_{n}, s_{n-1}\right) v\left(s_{n-1}\right), \\
\left\|v_{n}\right\|_{v} & \leq 2 \max \left(\sup _{r_{p_{n}} \leq r \leq r_{m_{n}}} M_{\infty}\left(v_{n}, r\right) v(r), \sup _{r_{m_{n}} \leq r \leq r_{q_{n}}} M_{\infty}\left(v_{n}, r\right) v(r)\right) \\
& \leq 2 \sqrt{b} M_{\infty}\left(v_{n}, r_{m_{n}}\right) v\left(r_{m_{n}}\right), \\
\left\|w_{n}\right\|_{v} & \leq 2 b M_{\infty}\left(w_{n}, s_{n}\right) v\left(s_{n}\right) .
\end{aligned}
$$

Since $f=u_{n}+v_{n}+w_{n}$ the result follows.

Combining Lemma 5.3 and Proposition 5.2 we obtain

5.4. Corollary. Assume that $b>4$. Then there are constants $c_{1}, c_{2}$ $>0$, indices $0 \leq k_{1} \leq k_{2} \leq \ldots$, radii $0<t_{1} \leq t_{2} \leq \cdots$ and uniformly bounded linear operators

$$
T_{n}: h v \rightarrow \operatorname{span}\left\{r^{|j|} \exp (i j \varphi): k_{n-2}<|j| \leq k_{n+1}\right\}
$$

satisfying the following.

For every trigonometric polynomial $f$ we have $f=\sum_{n} T_{n} f$,

$$
c_{1} \sup _{n} M_{\infty}\left(T_{n} f, t_{n}\right) v\left(t_{n}\right) \leq\|f\|_{v} \leq c_{2} \sup _{n} M_{\infty}\left(T_{n} f, t_{n}\right) v\left(t_{n}\right)
$$

and $T_{m} T_{n} f=0$ if $|n-m|>4$.

Finally,

$$
\|h\|_{v} \leq c_{2} M_{\infty}\left(h, t_{n}\right) v\left(t_{n}\right) \quad \text { whenever } h \in T_{n} h v, n=1,2, \ldots
$$


6. The Banach space geometry of $h v$ and $H v$. First we show 6.1. LEMMA.

(a) Let $m, n, p \in \mathbb{Z}_{+}$with $m \leq n \leq p$. Then $H_{m}$ is isometrically isomorphic to a 2-complemented subspace of $\left(H_{n} \oplus H_{p}\right)_{\infty}$.

(b) Consider integers $0<m<n$ and let $B_{n, m}=\operatorname{span}\left\{r^{|j|} \exp (i j \varphi)\right.$ : $j \in \mathbb{Z}$ and $m \leq|j| \leq n\}$ be endowed with the norm $M_{\infty}(\cdot, 1)$. Then there is an integer $N>0$ such that $B_{n, m}$ is isometrically isomorphic to a 16-complemented subspace of $\left(H_{N} \oplus H_{N}\right)_{\infty}$.

Proof. (a) For a complex function $f$ put $(W f)(z)=f(\bar{z})$. Identify $z^{j} \in$ $H_{m}$ with $\left(z^{j}, z^{m-j}\right) \in\left(H_{n} \oplus H_{p}\right)_{\infty}$. Put

$$
P(f, g)=\left(V_{m, 0} f+z^{m} W V_{m, 0} g, V_{m, 0} g+z^{m} W V_{m, 0} f\right) .
$$

Then $P$ is a projection from $\left(H_{n} \oplus H_{p}\right)_{\infty}$ onto $\left\{\left(z^{j}, z^{m-j}\right): j=0,1, \ldots, m\right\}$, which is isometrically isomorphic to $H_{m}$. We have $\|P\| \leq 2$.

(b) If $n \leq 2 m$, then, according to Lemma 3.3, the Riesz projection $R: B_{n, m} \rightarrow z^{m} H_{n-m}$ satisfies $\left\|\left.R\right|_{B_{n, m}}\right\|_{\infty} \leq 2$. Hence it follows that $d\left(B_{n, m},\left(H_{n-m} \oplus H_{n-m}\right)_{\infty}\right) \leq 4$, which yields (b) with $N=n-m$.

If $2 m<n$, then Lemma 3.3 implies $\left\|V_{2 n, n+m}\right\|_{\infty} \leq(n-m)^{-1}(3 n+m)$ $\leq 7$. Let $W$ be as in (a). Consider the space

$$
A=\operatorname{span}\left\{z^{j}: j \in \mathbb{Z}_{+}, 0 \leq j \leq n-m \text { or } n+m \leq j \leq 2 n\right\},
$$

endowed with the norm $M_{\infty}(\cdot, 1)$, which is isometrically isomorphic to $B_{n, m}$. Define $P:\left(H_{2 n} \oplus H_{2 n}\right)_{\infty} \rightarrow\left(H_{2 n} \oplus H_{2 n}\right)_{\infty}$ by

$$
\begin{aligned}
= & \left(V_{n-m, 0} f+\left(\mathrm{id}-V_{2 n, n+m}\right) f+z^{2 n} W V_{n-m, 0} g+z^{2 n} W\left(\mathrm{id}-V_{2 n, n+m}\right) g,\right. \\
& \left.V_{n-m, 0} g+\left(\mathrm{id}-V_{2 n, n+m}\right) g+z^{2 n} W V_{n-m, 0} f+z^{2 n} W\left(\mathrm{id}-V_{2 n, n+m}\right) f\right)
\end{aligned}
$$

We easily check that $P$ is a projection onto

$$
\operatorname{span}\left\{\left(z^{j}, z^{n+m-j}\right): j \in \mathbb{Z}_{+}, 0 \leq j \leq n-m \text { or } n+m \leq j \leq 2 n\right\},
$$

which is isometrically isomorphic to $A$. (Observe that $0 \leq j \leq n-m$ if and only if $n+m \leq 2 n-j \leq 2 n$.) We obtain $\|P\| \leq 16$, which proves (b) with $N=2 n$.

6.2. Corollary. Consider integers $0<m_{k} \leq n_{k}$ with $\lim _{k \rightarrow \infty}\left(n_{k}-m_{k}\right)$ $=\infty$ and let $B_{k}=\operatorname{span}\left\{r^{|j|} \exp (i j \varphi): j \in \mathbb{Z}_{+}\right.$and $\left.m_{k} \leq|j| \leq n_{k}\right\}$ be endowed with $M_{\infty}(\cdot, 1)$. Then

$$
\left(\sum_{k} \oplus H_{n_{k}}\right)_{\infty} \sim\left(\sum_{k} \oplus B_{k}\right)_{\infty} \sim H_{\infty}
$$

Proof. Put $X=\left(\sum_{m} \oplus H_{m}\right)_{\infty}$. Then $X$ is isomorphic to $H_{\infty}([22])$. Moreover, put $Y=\left(\sum_{k} \oplus H_{n_{k}}\right)_{\infty}$. We conclude that $Y$ is complemented 
in $X$. Using Lemma 6.1(a) we see that $X$ is complemented in $Y$. Since $H_{\infty} \sim$ $\left(H_{\infty} \oplus H_{\infty} \oplus \ldots\right)_{\infty}([22])$ this shows that $Y \sim H_{\infty}$. Using Lemma 6.1(a) we also see that every $H_{m}$ is 2-complemented in $\left(B_{k} \oplus B_{k^{\prime}}\right)_{\infty}$ for suitable $k$ and $k^{\prime}$. Hence $\left(\sum_{k} \oplus B_{k}\right)_{\infty}$ contains a complemented subspace isomorphic to $H_{\infty}$. Finally, Lemma $6.1(\mathrm{~b})$ implies that $\left(\sum_{k} \oplus B_{k}\right)_{\infty}$ is complemented in $H_{\infty}$. Hence $\left(\sum_{k} \oplus B_{k}\right)_{\infty} \sim H_{\infty}$.

6.3. Proposition. For any weight $v$ the spaces $h v$ and $H v$ are isomorphic to complemented subspaces of $H_{\infty}$, while $(h v)_{0}$ and $(H v)_{0}$ are isomorphic to complemented subspaces of $\left(\sum_{n} \oplus H_{n}\right)_{0}$.

Proof. Let $c_{1}, c_{2}, k_{m}, t_{m}$ and $T_{n}: h v \rightarrow \operatorname{span}\left\{r^{|j|} \exp (i j \varphi): k_{n-2}<\right.$ $\left.|j| \leq k_{n+1}\right\}=: B_{n}$ be as in Corollary 5.4, where $B_{n}$ is endowed with $\|\cdot\|_{v}$. Put $X=\left(\sum_{n} \oplus\left(B_{n},\|\cdot\|_{v}\right)\right)_{\infty}$. Define $U: X \rightarrow h v$ by $U\left(h_{n}\right)=\sum_{n} h_{n}$. Then, according to Corollary 5.4, $U$ is bounded. Indeed, we have $T_{m} h_{n}=0$ if $|n-m|>4$ and

$$
\left\|U\left(h_{n}\right)\right\|_{v} \leq c_{2} \sup _{m} M_{\infty}\left(T_{m} \sum_{n} h_{n}, t_{m}\right) v\left(t_{m}\right) \leq 6 c_{2}^{2} \sup _{n}\left\|h_{n}\right\|_{v} .
$$

Conversely, define $V: h v \rightarrow X$ by

$$
V f=\left(T_{n} f\right)_{n=1}^{\infty} .
$$

We have $\|V\| \leq c_{1}^{-1}$ and $U V=\mathrm{id}_{h v}$, which implies that $h v$ is isomorphic to a complemented subspace of $X$.

If $\sup _{n}\left(k_{n+1}-k_{n-2}\right)<\infty$, then $\sup _{n} \operatorname{dim} B_{n}<\infty$ and hence $\left(\sum_{n} \oplus B_{n}\right)_{\infty}$ $\sim l_{\infty}$. Since $l_{\infty}$ is complemented in $H_{\infty}$ the assertion of Proposition 6.3 follows.

If $\sup _{n}\left(k_{n+1}-k_{n-2}\right)=\infty$, then in view of Corollary 5.4 we have

$$
\sup _{n} d\left(\left(B_{n},\|\cdot\|_{v}\right),\left(B_{n}, M_{\infty}(\cdot, 1)\right)\right)<\infty
$$

(since $\left(B_{n}, M_{\infty}\left(\cdot, t_{n}\right) v\left(t_{n}\right)\right)$ is isometrically isomorphic to $\left.\left(B_{n}, M_{\infty}(\cdot, 1)\right)\right)$. We conclude, by Corollary 6.2 , that $X=\left(\sum_{n} \oplus B_{n}\right)_{\infty}$ is isomorphic to $H_{\infty}$. Again, the assertion follows in this case.

The proof for $H v$ instead of $h v$ is identical. Here, instead of $B_{n}$, we consider $\operatorname{span}\left\{r^{j} \exp (i j \varphi): k_{n-2}<j \leq k_{n+1}\right\}$, which is isometrically isomorphic to $H_{k_{n+1}-k_{n-2}-1}$.

Also the proof for $(H v)_{0}$ and $(h v)_{0}$ instead of $H v$ and $h v$ is identical.

Corollary 4.5 and Proposition 6.3 together with the decomposition method ([12]) prove Theorems 1.1(b) and 1.2(b). Theorems 1.1(a), 1.2(a) and 1.3 follow from

6.4. Proposition. Let $v$ satisfy (B). Then $\mathrm{Hv}$ and hv are isomorphic to $l_{\infty}$, while $(H v)_{0}$ and $(h v)_{0}$ are isomorphic to $c_{0}$. Moreover, the Riesz projection $R: h v \rightarrow H v$ is bounded. 
Proof. Let $m_{n}$ be the numbers of Lemma 5.1 with respect to some $b>2$. Then, using (B) and Proposition 4.1, we obtain universal constants $\eta, \kappa$ and $c, d$ such that $m_{n+1}-m_{n-1} \leq c$ or

$$
\eta \leq \frac{\left[m_{n+1}\right]-\left[m_{n}\right]}{\left[m_{n}\right]-\left[m_{n-1}\right]} \leq \kappa
$$

and

$$
\max \left(\left(\frac{r_{m_{n+1}}}{r_{m_{n-1}}}\right)^{m_{n+1}} \frac{v\left(r_{m_{n+1}}\right)}{v\left(r_{m_{n-1}}\right)},\left(\frac{r_{m_{n-1}}}{r_{m_{n+1}}}\right)^{m_{n-1}} \frac{v\left(r_{m_{n-1}}\right)}{v\left(r_{m_{n+1}}\right)}\right) \leq d
$$

for all $n$ with $m_{n-1} \geq c$. To prove the proposition it suffices to consider only those $n$ with $m_{n-1} \geq c$.

Put $T_{n}=V_{m_{n+1}, m_{n}}-V_{m_{n}, m_{n-1}}$. By (6.1), Lemma 3.3(c), (d) the operators $T_{n}$ are uniformly bounded with respect to $M_{\infty}(\cdot, 1)$ and hence with respect to $\|\cdot\|_{v}$ and to the norms $M_{\infty}\left(\cdot, r_{m_{n}}\right) v\left(r_{m_{n}}\right)$. From Corollary 3.2 we deduce

$$
\begin{aligned}
\left\|T_{n} h\right\|_{v} & \leq 2 d M_{\infty}\left(T_{n} h, r_{m_{n+1}}\right) v\left(r_{m_{n+1}}\right) \\
& \leq 2 d\left(\sup _{n}\left\|T_{n}\right\|_{\infty}\right) M_{\infty}\left(h, r_{m_{n+1}}\right) v\left(r_{m_{n+1}}\right)
\end{aligned}
$$

whenever $h \in h v$.

Let $Y_{n}$ be the space of all harmonic functions on $r_{m_{n+1}} D$ whose radial limits are $L_{\infty}$-functions on $\left\{z \in \mathbb{C}:|z|=r_{m_{n+1}}\right\}$. On $Y_{n}$ we consider the norm $M_{\infty}\left(\cdot, r_{m_{n+1}}\right) v\left(r_{m_{n+1}}\right)$ which is equivalent to $M_{\infty}\left(\cdot, r_{m_{n+1}}\right)$. Hence $Y_{n}$ is isometrically isomorphic to $L_{\infty}$. Note that the operators $V_{m, \widetilde{m}}$ make sense on $Y_{n}$ and $V_{m, \widetilde{m}} h$ is a trigonometric polynomial for every $h \in Y_{n}$.

If $m_{n+1}-m_{n-1}>c$ find finite-dimensional subspaces $X_{n} \subset Y_{n}$ with

$$
V_{m_{n+2}, m_{n+1}} Y_{n} \subset X_{n}
$$

and $\sup _{n} d\left(X_{n}, l_{\infty}^{\operatorname{dim} X_{n}}\right)<\infty$. If $m_{n+1}-m_{n-1} \leq c$ take $X_{n}=T_{n} h v$. Then $\operatorname{dim} X_{n} \leq c$. Altogether we obtain $\left(\sum_{n} \oplus X_{n}\right)_{0} \sim\left(\sum_{n} \oplus l_{\infty}^{\operatorname{dim} X_{n}}\right)_{0} \sim c_{0}$.

Define $U:\left(\sum_{n} \oplus X_{n}\right)_{0} \rightarrow(h v)_{0}$ by $U\left(h_{k}\right)=\sum_{k} T_{k} h_{k}$. (The functions $T_{k} h_{k}$ are trigonometric polynomials and therefore can be regarded as elements of $h v$.) Since $T_{n} T_{m}=0$ if $|n-m| \geq 2$ we have

$$
T_{n} U\left(h_{k}\right)=T_{n} T_{n-1} h_{n-1}+T_{n}^{2} h_{n}+T_{n} T_{n+1} h_{n+1} .
$$

Hence $\left\|T_{n} U\left(h_{k}\right)\right\|_{v} \leq c_{1} \sup _{j=n-1, n, n+1}\left\|T_{j} h_{j}\right\|_{v}$ for a universal constant $c_{1}$. Proposition $5.2,(6.2)$ and the uniform boundedness of the $T_{n}$ imply that $U$ is bounded.

If $m_{n+1}-m_{n-1} \leq c$ define, for $f=\sum_{k} \alpha_{k} r^{|k|} \exp (i k \varphi)$,

$$
S_{n} f=\sum_{m_{n-1}<|k| \leq m_{n+1}} \alpha_{k} r^{|k|} \exp (i k \varphi) \in X_{n} .
$$

Otherwise put $S_{n}=\left(\mathrm{id}-V_{m_{n-2}, m_{n-2} / 2}\right) V_{m_{n+2}, m_{n+1}}$. Define $V:(h v)_{0} \rightarrow$ $\left(\sum_{n} \oplus X_{n}\right)_{0}$ by $V f=\left(S_{n} f\right)$, which makes sense in view of (6.3). Recall that, 
since $b>2$ in view of Proposition 3.4, we have $\sup _{n}\left\|V_{m_{n+2}, m_{n+1}}\right\|_{v}<\infty$. Therefore, $V$ is bounded. Moreover, $U V f=\sum_{n} T_{n} f=f$. This implies that $(h v)_{0}$ is isomorphic to a complemented subspace of $\left(\sum_{n} \oplus X_{n}\right)_{0} \sim c_{0}$ and hence $(h v)_{0} \sim c_{0}([12])$. In view of Proposition 5.2, (6.1) and Lemma 3.3 the Riesz projection $R:(h v)_{0} \rightarrow(H v)_{0}$ is bounded. As a consequence we also have $(H v)_{0} \sim c_{0}$.

To prove the result for $h v$ instead of $(h v)_{0}$ we proceed exactly as before. Define $U:\left(\sum_{n} \oplus X_{n}\right)_{\infty} \rightarrow h v$ by $U\left(h_{k}\right)=\sum_{k} T_{k} h_{k}$. From Proposition 5.2 and (6.2), looking at the Fourier series, we see that the series $\sum_{k} T_{k} h_{k}$ converges pointwise to a harmonic function (called $\sum_{k} T_{k} h_{k}$ again) with $\left\|\sum_{k} T_{k} h_{k}\right\|_{v}<\infty$. Hence $\sum_{k} T_{k} h_{k} \in h v$. The definition of $V$ can be repeated literally for the operator $h v \rightarrow\left(\sum_{n} \oplus X_{n}\right)_{\infty}$ with $U V=\operatorname{id}_{h v}$. Hence we obtain $h v \sim l_{\infty}$ and the Riesz projection $R: h v \rightarrow H v$ is bounded. Therefore we also have $H v \sim l_{\infty}$. (Alternatively, we could have used Proposition 5.2 or $[1,18]$ to see that $h v \sim(h v)_{0}^{* *} \sim l_{\infty}$ and $H v \sim(H v)_{0}^{* *} \sim l_{\infty}$.)

\section{References}

[1] K. D. Bierstedt and W. H. Summers, Biduals of weighted Banach spaces of analytic functions, J. Austral. Math. Soc. Ser. A 54 (1993), 70-79.

[2] O. Blasco, Multipliers on weighted Besov spaces of analytic functions, in: Contemp. Math. 144, Amer. Math. Soc., 1993, 23-33.

[3] J. Bonet and E. Wolf, A note on weighted Banach spaces of holomorphic functions, Arch. Math. (Basel) 81 (2003), 650-654.

[4] P. Domański and M. Lindström, Sets of interpolation and sampling for weighted Banach spaces of holomorphic functions, Ann. Polon. Math. 89 (2002), 233-264.

[5] P. L. Duren, Theory of $H^{p}$ Spaces, Academic Press, New York, 1970.

[6] T. M. Flett, The dual of an inequality of Hardy and Littlewood and some related inequalities, J. Math. Anal. Appl. 38 (1972), 746-765.

[7] —, Lipschitz spaces of functions on the circle and the disc, J. Math. Anal. Appl. 39 (1972), 125-158.

[8] A. Galbis, Weighted spaces of entire functions, Arch. Math. (Basel) 62 (1994), 58-64.

[9] D. J. H. Garling and P. Wojtaszczyk, Some Bargmann spaces of analytic functions, in: Function Spaces (Edwardsville, IL, 1994), Lecture Notes in Pure and Appl. Math. 172, Dekker, 1995, 123-138.

[10] J. Hilsmann, Struktursätze für Banachräume holomorpher Funktionen in mehreren Variablen, Doctoral Dissertation, Univ. of Dortmund, 1985.

[11] K. Hoffman, Banach Spaces of Analytic Functions, Prentice-Hall, Englewood Cliffs, NJ, 1962.

[12] J. Lindenstrauss and L. Tzafriri, Classical Banach Spaces I, Springer, Berlin, 1986.

[13] W. Lusky, On weighted spaces of harmonic and holomorphic functions, J. London Math. Soc. 59 (1995), 309-320.

[14] —, On generalized Bergman spaces, Studia Math. 119 (1996), 77-95.

[15] - On the Fourier series of unbounded harmonic functions, J. London Math. Soc. (2) 61 (2000), 568-580. 
[16] W. Lusky, On the isomorphic classification of weighted spaces of holomorphic functions, Acta Univ. Carolin. Math. Phys. 41 (2000), 51-60.

[17] M. Mateljević and M. Pavlović, $L^{p}$-behaviour of the integral means of analytic functions, Studia Math. 77 (1984), 219-237.

[18] L. A. Rubel and A. L. Shields, The second duals of certain spaces of analytic functions, J. Austral. Math. Soc. 11 (1970), 276-280.

[19] A. L. Shields and D. L. Williams, Bounded projections, duality and multipliers in spaces of analytic functions, Trans. Amer. Math. Soc. 162 (1971), 287-302.

[20] - - - Bounded projections, duality and multipliers in spaces of harmonic functions, J. Reine Angew. Math. 299/300 (1978), 256-279.

[21] - - - Bounded projections and the growth of harmonic conjugates in the unit disc, Michigan Math. J. 29 (1982), 3-25.

[22] P. Wojtaszczyk, On projections in spaces of bounded analytic functions with applications, Studia Math. 65 (1979), 147-173.

Fakultät für Elektrotechnik,

Informatik und Mathematik

Universität Paderborn

Warburger Straße 100

D-33098 Paderborn, Germany

E-mail: lusky@uni-paderborn.de

Received February 2, 2005

Revised version December 30, 2005 